\title{
Cellulose Derivative/Barium Titanate Composites with High Refractive Index, Conductivity and Energy Density
}

\section{Andreea Irina Barzic}

Institutul de Chimie Macromoleculara Petru Poni

Marius Soroceanu

Institutul de Chimie Macromoleculara Petru Poni

Razvan Rotaru

Institutul de Chimie Macromoleculara Petru Poni

Florica Doroftei

Institutul de Chimie Macromoleculara Petru Poni

Mihai Asandulesa ( $\square$ asandulesa.mihai@icmpp.ro )

Institutul de Chimie Macromoleculara Petru Poni https://orcid.org/0000-0002-3459-7015

Valeria Harabagiu

Institutul de Chimie Macromoleculara Petru Poni

\section{Research Article}

Keywords: polymer composites, refractive index, transparency

Posted Date: May 10th, 2021

DOl: https://doi.org/10.21203/rs.3.rs-467926/v1

License: (9) (i) This work is licensed under a Creative Commons Attribution 4.0 International License. Read Full License

Version of Record: A version of this preprint was published at Cellulose on January 9th, 2022. See the published version at https://doi.org/10.1007/s10570-021-04343-2. 
1 CELLULOSE DERIVATIVE/BARIUM TITANATE COMPOSITES WITH HIGH REFRACTIVE INDEX, CONDUCTIVITY AND ENERGY DENSITY

3

4 5 6

Andreea Irina Barzic, Marius Soroceanu, Razvan Rotaru, Florica Doroftei, Mihai Asandulesa*, Valeria Harabagiu

"Petru Poni” Institute of Macromolecular Chemistry, 41A Grigore Ghica Voda Alley, 700487 Iasi, Romania

Abstract: High refractive index and transparent materials are useful in various technical domains, ranging from energy sector to microelectronics. This work deals with polymer composites prepared by embedding small amounts of barium titanate in hydroxypropyl cellulose matrix. Optical transparency of the composite films varies in agreement with polymer doping level. Light dispersion in the prepared composites allowed evaluation of specific parameters, which are related to the sample structural order and possible optical transitions. Conductivity of the composites is increased due to ceramic particle addition in the polymer. Dielectric studies reveal that the prepared materials are suitable for electric energy storage applications.

Keywords: polymer composites, refractive index, transparency

(1)

2

33

4

5

\footnotetext{
* Correspondence to: M. Asandulesa; e-mail: asandulesa.mihai@icmpp.ro
} 


\section{INTRODUCTION}

In the global context of resource lessening, energy increasing demands and planet pollution, the topics of polymer science are more and more concerned with replacing parts of high-tech products with others that are striving to match the demands of low-carbon society (Zhang et al., 2019). Hence, in order to alleviate the environmental burden, it is imperative to develop biodegradable, less toxic and advanced materials for many technical areas, like high power electronics and energy storage. In the past years, film dielectric capacitors with high energy density opened fresh perspectives due to lightweight, flexible, and elevate power densities (i.e. rapid discharge of stored electric energy). For such purposes, the performance of the material can be controlled via dielectric constant and breakdown strength by combining plastics with inorganic fillers.

Barium titanate (BT) is an inorganic lead-free ferroelectric compound, which is well known in materials science for its photorefractive and piezoelectric properties (Takenaka, 2013). Other essential characteristics of this ceramic material are: high polarization $\left(\sim 26 \mu \mathrm{C} \mathrm{cm}^{-2}\right)$ (Takenaka, 2013) and elevated permittivity (100-2000) (Petrovsky et al., 2008), quite large band gap of $3.2 \mathrm{eV}$ (Yogamalar et al., 2018), and relatively high piezoelectric strain constant ( $190 \mathrm{pC} / \mathrm{N}$ ) (Yogamalar et al., 2018; Zhang et al., 2019). Since most of these features are rarely encountered in polymer materials, BT is widely used as filler for fabrication of polymer micro/nano-composites. Therefore, the utility of BT-reinforced polymers has expanded very fast in various areas of technology and industry. Among them applications in energy storage (Yao et al., 2018; Yue et al., 2019), sensors (Yang et al., 2020), embedded capacitors (Goyal et al., 2013), high refractive (Nagao et al., 2011) and nonlinear optics coatings, are included (Mimura et al., 2010). The extent of the modification of the polymer properties by BT filler is affected by the level of its dispersion inside the matrix as well as by the size of the BT particles (Gu et al., 2019). An essential aspect of such composites is related to the possibility to upgrade the desired properties at very small filler loadings inserted in the polymer phase (Sen et al., 2016; Yue et al., 2019).

Several types of polymer matrices were used for preparation of BT-reinforced micro/nanocomposites as a function of desired balance of properties imposed by application. BT/epoxy resin films were synthesized via a complex alkoxide procedure and showed a dielectric constant of 10.8 at 30\% filler (Kobayashi et al., 2010). Poly(vinylidene fluoride-trifluoroethylene)/BT composites were analyzed by polarization-electric field method, showing improved ferroelectric properties, which are suitable for nonvolatile memory devices (Valiyaneerilakkal et al., 2015). Nanocomposites containing BT filler were prepared by solvent casting and by using as matrix a blend of 
poly(ethylene oxide) and poly(vinyl pyrrolidone) doped with $\mathrm{LiClO}_{4}$ (Kesavan et al., 2015). The increase of ceramic filler amount has positively influenced the ionic conductivity and the thermal stability as demanded by lithium battery applications (Kesavan et al., 2015). Polyethylene oxide/BT/LiClO 4 composites, analyzed by impedance spectroscopy, revealed a conductivity of 7.6 $10^{-6} \mathrm{~S} / \mathrm{cm}$ owing to the powerful interactions between polymer and ceramic nanoparticles (Malathi et al., 2015). Such materials are candidates for solid-state polymer batteries and fuel cells. BT nanoparticles were also inserted in ethyl cellulose up to $13 \mathrm{vol} \%$, leading to materials with enhanced dielectric constant $(\sim 7.5$ at $1 \mathrm{~Hz})$, high dielectric loss and raised de conductivity. At low amount of reinforcements and below $60^{\circ} \mathrm{C}$, the composites are good for antistatic applications, whereas above $7 \mathrm{vol} \%$ of BT and at high temperatures the films are suitable for electrostatic dissipation purposes (Ali et al., 2017). BT-acrylic hybrid materials produced by photopolymerization were shown to display dielectric constants that range almost linearly with nanoparticle loading up to 76 at $1 \mathrm{kHz}$ at $50 \mathrm{wt} \%$ filler (Lombardi et al., 2011). The dielectric properties of these composites are adequate for capacitor applications. Olmos and co-workers (Olmos et al., 2013) reported the influence of processing conditions and of BT filler amount on morphology of polyvinylidene fluoride, proving that the milling process is the main factor to enhance the percent of $\beta$-phase being advantaged by the presence of BT particles. Also, the matrix lamellar aspect ratio is enhanced by the amount of filler (Olmos et al., 2013). Piezoelectric polyamide-11/BT composites were achieved by using solid-state mechanochemical procedure (Hua et al., 2019). A linear dependence was found between piezoelectric strain coefficient and filler loading, reaching $6.6 \mathrm{pC} / \mathrm{N}$ at $50 \mathrm{vol} \% \mathrm{BT}$. These composites displayed good mechanical resistance, being ideal for sensor applications. Composites with high permittivity were prepared by adding BT in polyimide and processing by electrospinning (Xu et al., 2016). The resulted hybrid nanofiber sheets reached a dielectric constant of 29.7 when the percent of BT was $50 \mathrm{vol} \%$, being adequate for embedded capacitors or energy-storage uses (Xu et al., 2016). Other studies were focused on preparation of multiphase polymer nanocomposites by addition of BT and other kinds of fillers. Thus, polyimide/ $\mathrm{SiO}_{2}-\mathrm{BT}$ composites were proved to have high thermal stability (Gao et al., 2008). Ternary $\mathrm{NiNb}_{2} \mathrm{O}_{6} / \mathrm{BT} /$ poly(arylene ether nitriles) composites obtained via solution casting technique, showed great improvements at $40 \mathrm{wt} \%$ filler in terms of tensile properties and dielectric constant ( $\sim 15.3$ at $1 \mathrm{kHz}$ ), being suitable candidates for organic capacitors (Huang et al., 2020). Most reported studies are focused on structural, morphological, thermal and dielectric properties of BT-polymer composites and very few address aspects related to their optical properties (Mimura et al., 2010; Nagao et al., 2011; Morsi et al., 2019). This is probably because, at high BT loadings, the transparency of the material is compromised and limits the possibility of material investigation. 
Optical absorption features, particularly the absorption edges are essential for describing optical transitions, the band structure, the band tail and the band gap energies of composite materials. To our knowledge, only the study of Morsi et al. (Morsi et al., 2019) describes optical constants of BT composites with polyethylene oxide/carboxymethyl cellulose (PEO/CMC) as matrix. Based on optical and electrical data they show that such materials can be used for sensors, electromechanical transducers or as ceramic capacitors. On the other hand, Nagao et al. (Nagao et al., 2011) demonstrates that the incorporation of BT in poly(methyl methacrylate) increases both the refractive index and the permittivity of the composites, with potential use in displays and other electronic devices.

Previously, we have prepared viscose fiber/BT composites of low BT content and proved the appropriateness of their properties for applications as absorbent materials in electromagnetic fields (Rotaru et al., 2016). In this work, new BT composites with biodegradable hydroxypropyl cellulose (HPC) matrix were prepared. Structural, optical and electrical properties were analyzed to demonstrate their usefulness for energy storage. The performed investigations will prove that the HPC/BT materials have higher refractive index than PMMA/ethylene glycol monovinyl ether/BT (Mimura et al., 2010) as well as larger permittivity and conductivity in comparison with PEO/CMC/BT (Morsi et al., 2019) and polypropylene/BT composites (Yao et al., 2018). Energy density evaluations reveal similar values to those reported for polyimide/BT composites (Yue et al., 2019).

\section{EXPERIMENTAL}

\subsection{Materials}

Klucel $^{\mathrm{TM}}$ Hydroxypropyl cellulose (HPC) of $100000 \mathrm{~g} / \mathrm{mol}$ molar mass and substitution degree of 2.5 was purchased from Aqualon Company.

Barium carbonate and titanium dioxide were purchased from Sigma Aldrich and used as received.

\subsection{Preparation of BT}

Barium titanate (BT) was prepared by using a wet-state reaction under ultrasonic irradiation. The detailed procedure is described in a previous work (Rotaru et al., 2017). Shortly, a 1/2 (w/w) titanium dioxide / barium carbonate mixture was ultrasonically irradiated at $20 \mathrm{kHz}$ frequency, in Milli-Q ultrapure water for 60 minutes $(119 \mathrm{~kJ}$ energy were dissipated and the temperature reached 
$92^{\circ} \mathrm{C}$ ) by using a generator equipped with probe and sensor for temperature. The obtained powder was further decanted and dried in a microwave furnace for 10 minutes. The last step was the thermal treatment for 3 hours, performed in a laboratory furnace at temperatures of $500^{\circ} \mathrm{C}$.

\subsection{Preparation of HPC/BT composite films}

Film sample preparation was done as follows: a stock polymer solution was obtained by dissolving $5 \mathrm{~g} \mathrm{HPC}$ in $250 \mathrm{ml}$ of deionized water at room temperature. $50 \mathrm{~mL}$ of HPC solution and known amounts of BT particles of different filler loadings $(0.5,1$ and $2 \%$ BT) were homogenized by ultrasonication for 10 minutes; during this procedure, $7 \mathrm{~kJ}$ energy was dissipated and the temperature increased from $20^{\circ} \mathrm{C}$ to $40^{\circ} \mathrm{C}$. The prepared dispersions were poured onto Petri dishes of $10 \mathrm{~cm}$ diameter. The films were obtained by drying overnight at room temperature and then they were kept in a vacuum oven at $90^{\circ} \mathrm{C}$ for 6 hours. The pure HPC film was obtained by using a similar procedure.

The thickness of the obtained HPC and composite films ranged between 70 to $140 \mu \mathrm{m}$ as measured with a digital micrometer.

\subsection{Characterization}

The structures of barium titanate (BT) powder, pristine HPC film and the HPC/BT composites films were investigated by FTIR spectroscopy in potassium bromide pellets using a BrukerVertex 70 Spectrometer.

In order to highlight the morphology of the samples, they were examined on the surface and in cross-section. Surface morphology for the control HPC sample and HPC/BT composites were analyzed on a Verios G4 UC scanning electron microscope (SEM) from Thermo Scientific, Czech Republic. The samples were coated with $10 \mathrm{~nm}$ platinum using a Leica EM ACE200 Sputter coater to render electrical conductivity and to obstruct charge buildup during exposure to the electron beam. SEM investigations were performed in high vacuum mode using a secondary electron detector (Everhart-Thornley detector, ETD) at accelerating voltage of $5 \mathrm{kV}$. Cross-sectional analysis of films was performed on a Quanta 200 device (FEI, Czech Republic). To achieve the cross section, the films were immersed in liquid nitrogen to impede deformation during fracture. The presence of the BT was confirmed by energy dispersive X-ray (EDX) analysis using the Quanta 200 microscope coupled with EDX detector.

UV-VIS features of the free-standing films were examined on a SPECORD 210 PLUS device. 
Refraction properties of film samples were measured on a DR-M4 Abbe refractometer at several wavelengths from visible range.

Dielectric measurements were carried out on a broadband dielectric spectrometer equipped with a high resolution Alpha-A analyzer. The dielectric spectra were recorded at room temperature, in a frequency range between $1 \mathrm{~Hz}$ and $10^{6} \mathrm{~Hz}$.

\section{RESULTS AND DISCUSSION}

HPC was used as matrix for the preparation of organic-inorganic composites of different BT contents. To obtain optically transparent composites a maximum of $2 \%$ of BT filler was used.

\subsection{FTIR analysis}

The structures of the precursors and the composites were first assessed through FTIR spectroscopy (Figure 1). As observed from Figure 1, HPC displays prominent bands at 1049, 1057, $1080 \mathrm{~cm}^{-1}$ corresponding to $-\mathrm{C}-\mathrm{O}-\mathrm{C}-$ stretching, while those noticed at $1643,3443 \mathrm{~cm}^{-1}$ and at $658,582,476$ $\mathrm{cm}^{-1}$ are assigned to $-\mathrm{OH}$ stretching and to out of plain bending of $\mathrm{C}-\mathrm{OH}$ units. The bands in the region 2880-2974 $\mathrm{cm}^{-1}$ and that centered at $1464 \mathrm{~cm}^{-1}$ are attributed to $\mathrm{C}-\mathrm{H}$ stretching, respectively to $\mathrm{C}-\mathrm{H}$ in-plane bending vibrations. Other spectral bands, such as, $1335 \mathrm{~cm}^{-1}\left(\mathrm{CH}_{2}\right.$ rocking vibration at C6), $1381 \mathrm{~cm}^{-1}$ (CH in-plane bending), $1418 \mathrm{~cm}^{-1}$ (HCH in plane bending vibration, $\mathrm{CH}_{2}$ scissoring motion, $\mathrm{CH}_{2}$ symmetric bending at $\mathrm{C} 6$ ) are also attributed to alkyl units of the cellulose chain (Sharma et al., 2009; Abdel-Halim et al., 2015; Joshi et al., 2019).

The infrared spectrum of BT shows characteristic bands at $438 \mathrm{~cm}^{-1}$ and $559 \mathrm{~cm}^{-1}$. The band observed at lower wavenumber $\left(438 \mathrm{~cm}^{-1}\right)$ is one of the "fingerprints" of BT and it is attributed to the $\mathrm{Ba}-\mathrm{O}$ and $\mathrm{Ti}-\mathrm{O}$ metal oxides vibration. The presence of the band at higher wave number (559 $\mathrm{cm}^{-1}$ ) is ascribed to $\mathrm{Ti}-\mathrm{O}_{\mathrm{I}}$ normal stretching vibration (characteristic to $\mathrm{BaTiO}_{3}$ tetragonal phase) (Rotaru et al., 2016). The FTIR spectrum of the composite with a content of $0.5 \%$ BT filler, shown as a typical one for all HPC/BT samples presents all characteristic bands of HPC matrix, while the "fingerprints" of the filler are superposed with $\mathrm{COH}$ out-of plane bending vibrations of HPC in a large and high intensity band, centered at $615 \mathrm{~cm}^{-1}$. 


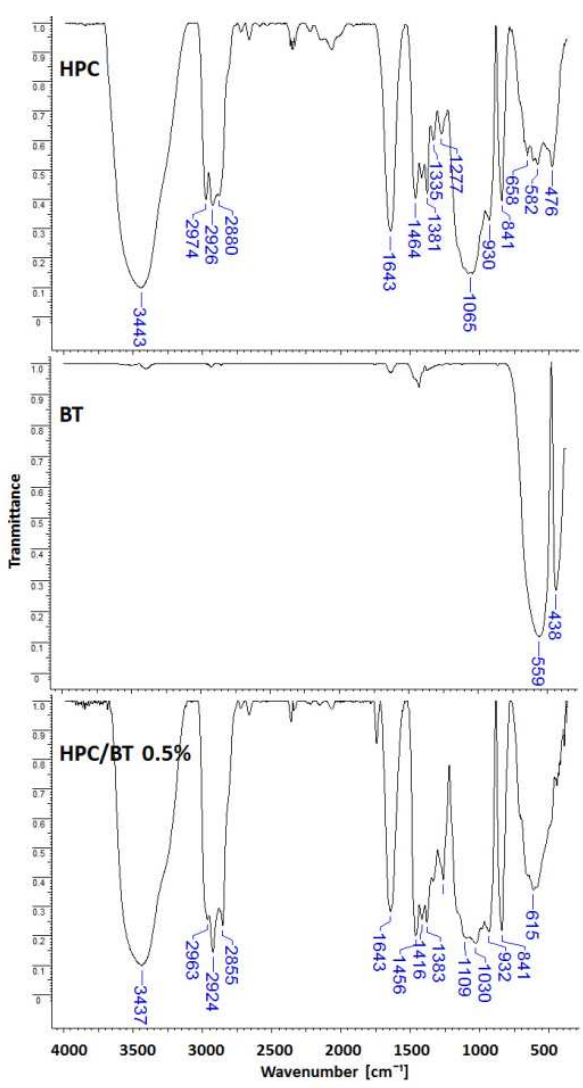

FIGURE 1 FTIR spectra of HPC film, BT powder, and HPC/BT 05\% composite films

\subsection{Morphology}

The morphological investigations were made by SEM coupled with EDX for the neat HPC and BT filled films. Figure 2 shows the surface micrographs and EDX compositional results. The HPC matrix displays mainly a relatively textured surface sprinkled with small defects probably formed due to partially crystalline nature of cellulose material (Vanderghem et al., 2012; Mohd Ishak et al., 2020). In a previous work (Rotaru et al., 2016), the morphology of the used BT powder was found to consist in agglomerated particles having almost spherical shape, while their diameters varied between 550-1300 nm. Here, these ceramic particles can be easily observed as white approximately spherical dots which are present for all filler concentrations and with increasing particles' density on the film surface with increasing BT content. The ceramic filler is well and uniformly dispersed within the cellulose derivative matrix on the surfaces of the samples. Relatively similar morphology was noted for BT/polyaniline composites (Pant et al., 2006). The qualitative results on film compositions provided by the EDX analysis are presented in the tables inserted in Figure 2. EDX recordings show the lack of $\mathrm{Ba}$ and Ti elements on the surface of pristine HPC, while, upon reinforcement, the percentage of these chemical elements is closed to the 
content of BT filler in each composite sample. EDX analysis is a volume analysis which means that the ceramic BT particles dispersion is uniform not only on the surface but also inside the reinforced polymer films.
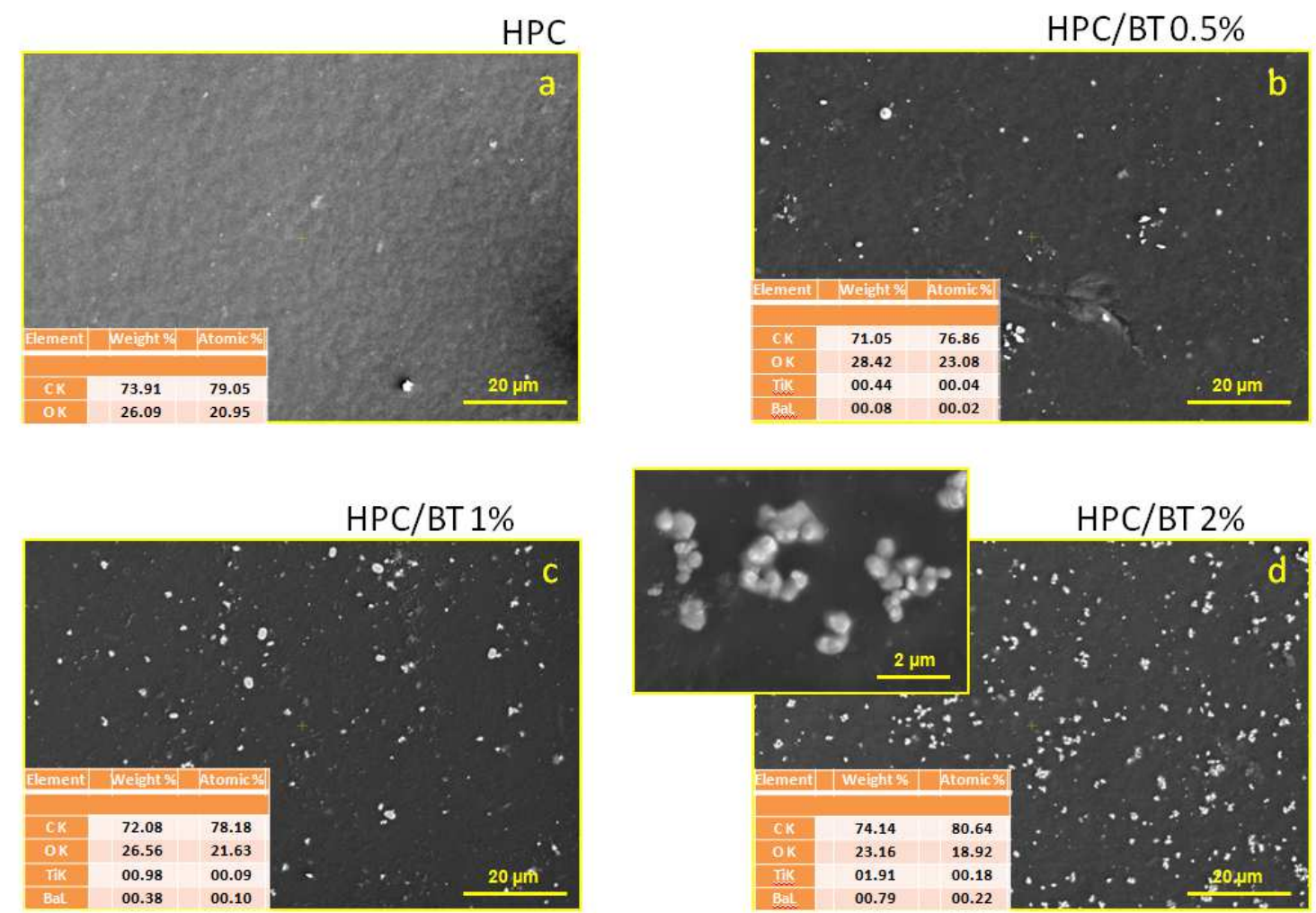

FIGURE 2 SEM micrographs of the surface of HPC and HPC/BT composites: (a) HPC, (b) HPC/BT 0.5\%, (c) HPC/BT 1\% and (d) HPC/BT 2\%

Cross-section SEM images of HPC and HPC/BT samples are depicted in Figure 3. HPC, known for its thermotropic liquid crystalline behavior generated a film which displayed a granular structure, with small pores, typical for a three-dimensional network (Nishio \& Takahashi, 1984; Silva et al., 2008). The incorporation of small amounts $(0.5,1 \%)$ of BT in the HPC matrix does not change this morphology. However, when $2 \%$ BT were added into HPC matrix, a pleated morphology of the cross-section of the resulted film was observed. Moreover, HPC/BT films show more compact morphology with a lower porosity as compared to the pristine HPC film, especially for HPC/BT 2\% sample, also evidenced by decreasing values of film thickness as a function of BT content (Table 1). Increased compactness of the composite films was earlier evidenced by Banerjee et al. (Banerjee et al., 2019) for poly(vinyl alcohol)/ manganese chloride composites. 
HPC

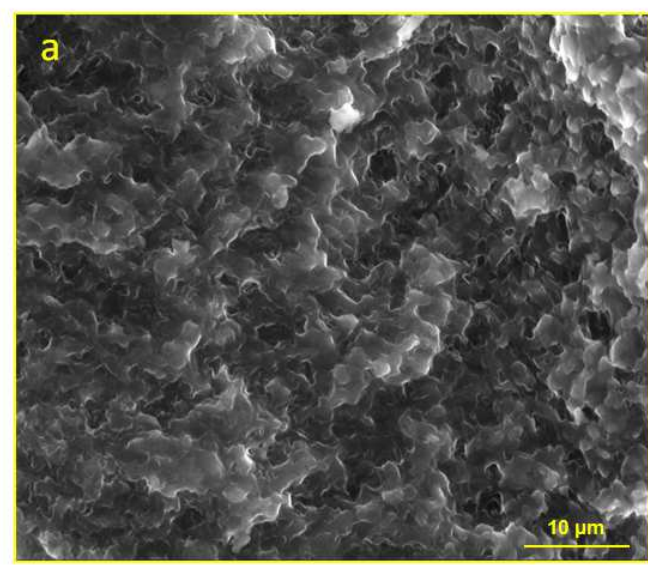

HPC/BT 1\%

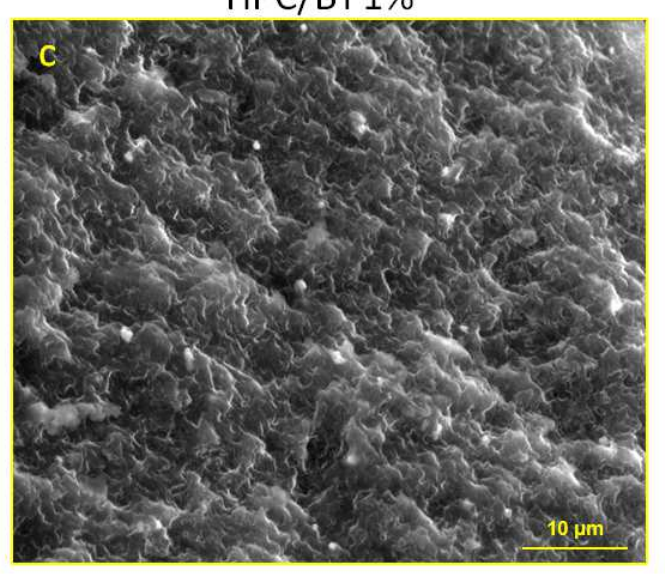

HPC/BT 0.5\%

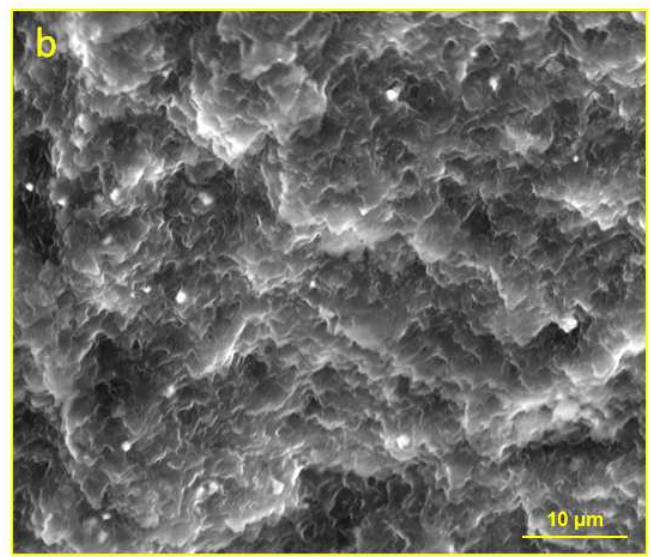

HPC/BT 2\%

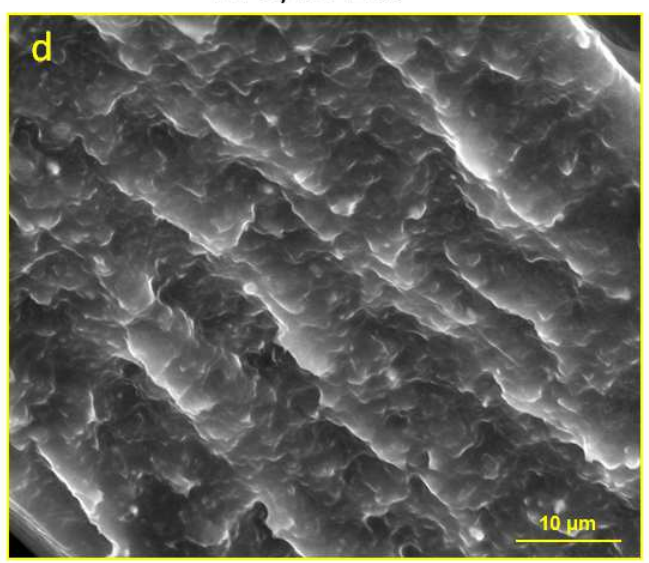

FIGURE 3 SEM micrographs: Cross-sections of HPC and HPC/BT composites films: (a) HPC, (b) HPC/BT $0.5 \%$, (c) HPC/BT 1\% and (d) HPC/BT 2\%

\subsection{Absorption edge and band gap energies}

The transparency properties of HPC and HPC/BT composite samples were investigated by UVVIS-NIR spectroscopy on free-standing films. Figures $4 \mathrm{a}$ and $\mathrm{b}$ show the spectra samples. A sharp absorption edge is noticed in the spectrum of the cellulosic matrix, which is slightly enhanced by the addition of BT particles in the HPC matrix. The observed absorbance behavior of the prepared composite samples presents changes in the magnitude of the optical band gap energy with further loading of BT filler in the system, reflecting the possible interactions between the two phases. Similar spectral features were reported by Morsi et al. (Morsi et al., 2019) for composites of PEO/CMC filled with BT particles. For the investigated samples, the optical clarity of the prepared materials is gradually diminished and the absorbance is enhanced as BT loading increases from $0 \%$ to $2 \%$ in the HPC matrix. Thus, the reinforcement of the composite films favors light scattering and enhances the absorption coefficient. This aspect can be observed from a simple visual examination 
of the free-standing films, since their aspect gradually changes from transparent to almost white color (Figure $4 \mathrm{~b}$ ). Absorption and scattering phenomena caused by $1 \%$ and $2 \%$ BT reinforcement of cellulose derivative are altering the transparency of pristine HPC film.

(a)

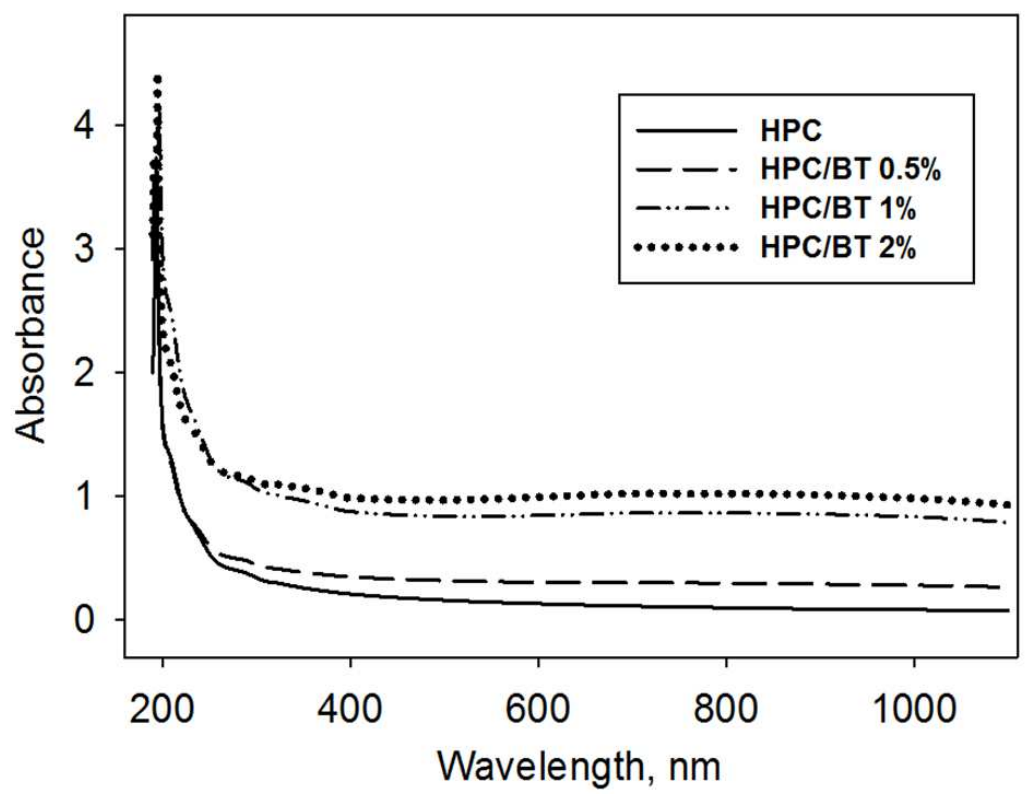

(b)

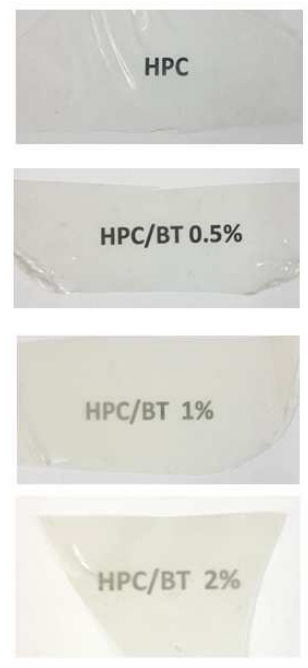

257

FIGURE 4 UV-VIS-NIR spectrum of the neat HPC and HPC/BT composite films (a) and the pictures of analyzed samples (b)

The optical absorption coefficient characterizes the material ability to absorb electromagnetic radiations, particularly from visible range. Optical absorption coefficient was estimated for each of the investigated samples by introducing UV-VIS data in equation (1):

$\alpha=\frac{1}{t} \ln \left(\frac{1}{T}\right)$

where $\alpha$ is the optical absorption coefficient, $t$ is the thickness of the film sample and $\mathrm{T}$ is the transmittance.

For all spectra illustrated in Figure 4a, the absorption edge, representing the low energy wing of the first absorption band, is analogous to the edge suggested by Tauc approach (Tauc, 1967) for ideal amorphous semiconductors. Given this similarity, the absorption edge parameters were determined from the dependence of absorption coefficient on photon energy, as depicted in equation (2):

$\alpha \sim \exp \frac{E}{E_{0}}$

where $\mathrm{E}$ is the photon energy $(\mathrm{E}=\mathrm{hv}), \mathrm{E}_{0}$ can be either Tauc energy (Et) or Urbach energy $(\mathrm{Eu})$.

Graphical representations of equation (2) as semi-logarithmic plots are following the Urbach relation as shown in Figure 5. Absorption edge energies were extracted from the reverse of slopes 
of the straight lines and the results are listed in Table 1. Et is the optical parameter extracted from the low-energy exponential zone of the dependence of absorption coefficient on photon energy, while $\mathrm{Eu}$ is related to the high-energy exponential region. For pristine HPC matrix, as well as for HPC/BT composites the values of Et are higher than those of Eu. Therefore, the absorption related to possible structural defects (i.e. break or torsion of polymer chains) is more pronounced with regard to absorption determined by structural disorder. No report on $\mathrm{Eu}$ and Et was found in literature for polymer/BT composites. Moreover, BT reinforcement generates intensification of these absorption processes as seen in the increase of Et and Eu parameters.

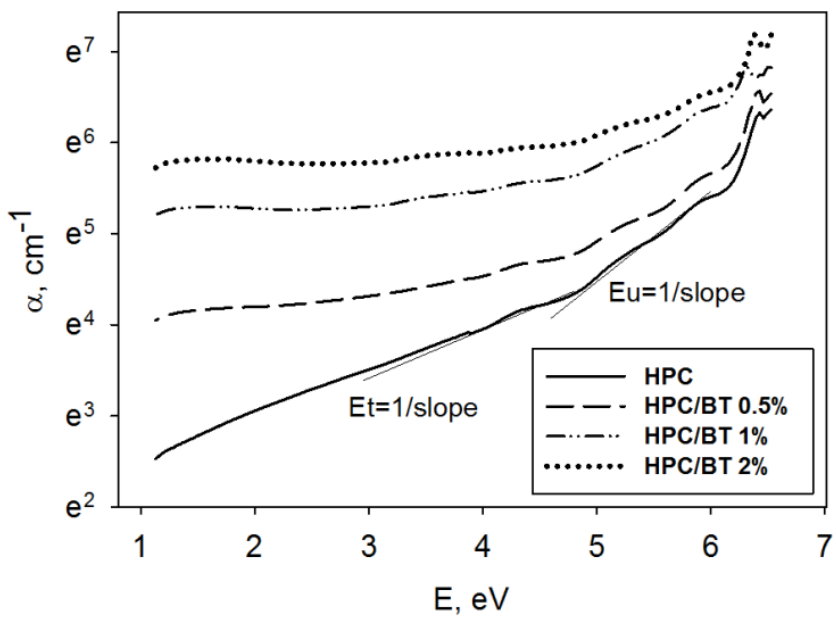

FIGURE 5 Optical absorption coefficient versus photon energy for unfilled HPC and HPC/BT composite films

TABLE 1 The values of the film thickness, Tauc energy (Et), Urbach energy (Eu), indirect band gap (Egi) and direct band gap (Egd) values for pristine HPC and HPC/BT composites

\begin{tabular}{|l|c|c|c|c|c|}
\hline Sample code & Thickness, $\mu \mathrm{m}$ & Et, meV & Eu, meV & Egi, eV & Egd, eV \\
\hline HPC & 140 & 2046 & 1166 & 5.71 & 6.12 \\
\hline HPC/BT 0.5\% & 110 & 3294 & 1363 & 5.43 & 6.03 \\
\hline HPC/BT 1\% & 100 & 4474 & 1570 & 5.31 & 5.96 \\
\hline HPC/BT 2\% & 70 & 6858 & 2181 & 5.20 & 5.81 \\
\hline
\end{tabular}

The photon energy that is demanded for transporting the electrons from the valence to the conduction band can be estimated by examining the region of the absorption boundary. Based on literature data (Choudhary \& Sengwa, 2018; Morsi et al., 2019; Sengwa et al., 2019), it is well known that the majority of photon absorbing materials are characterized by a direct and an indirect 
where $C$ is a constant and $m$ is a parameter that describes the nature of the electronic transitions, taking the value of 0.5 for direct allowed transitions and 2 for indirect allowed transitions.

Figure 6 displays the plots of $(\alpha E)^{1 / m}$ versus photon energy for $m=0.5$ and $m=2$. Linear parts are noticeable in all plots and their intersection with $\mathrm{x}$-axis is essential for establishing the Eg values for the prepared samples. Electrons travelling from the valence to the conduction band, while conserving total energy and the wave-vector k-space, denotes a direct transition. On the other side, electron moving in the conduction band at distinct k-spaces produces an indirect transition. The estimated values of indirect band gap (Egi) and direct band gap (Egd) for the investigated samples are illustrated in Table 1. Regardless the filling level of HPC, the results indicate lower values for Egi as compared to Egd. Moreover, the reduction of optical band gap energy with enhancement of inorganic filler loading was registered, a common feature for polymer composites (Choudhary \& Sengwa, 2018; Morsi et al., 2019; Sengwa et al., 2019). The obtained band gap values for HPC/BT are slightly higher than those reported by Morsi et al (Morsi et al., 2019) for PEO/CMC filled with BT. The presence of ceramic BT particles within HPC matrix facilitates electron crossing in the valence band with production of supplementary localized energy states in the forbidden energy zone and therefore lowering the band gap of the BT/HPC materials. The Eg reduction for composites with the variation in content of the constituting phases is believed to enhance with the degree of disorder (higher $\mathrm{Eu}$ ) in the material, which determines an increase in the flexibility of the macromolecular chains. Hence, the charge carriers, particularly at higher reinforcement degrees have better mobility and the electrical features will improve.

(a)

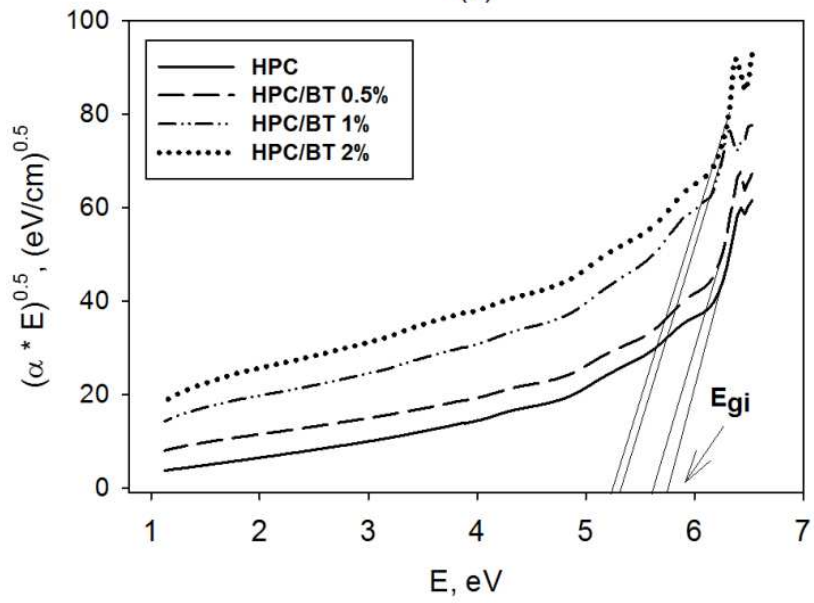

(b)

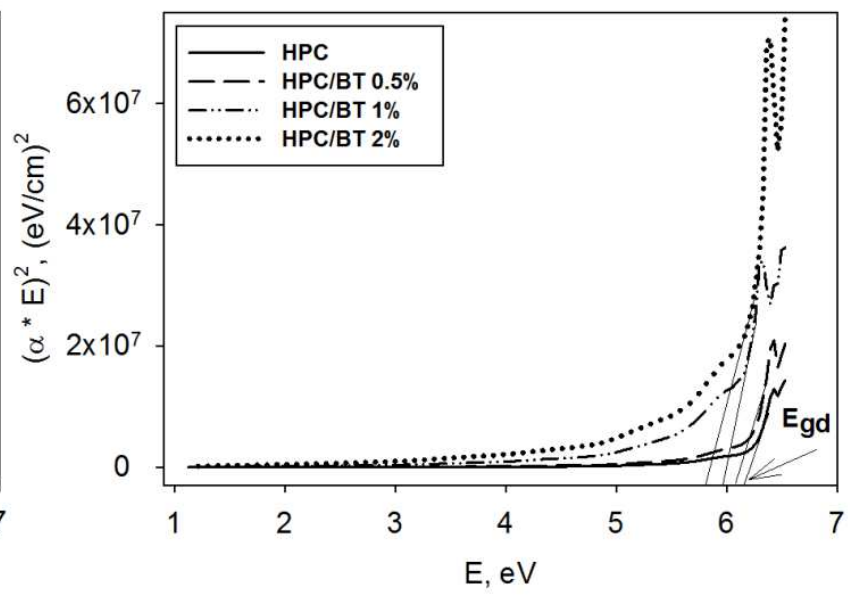


FIGURE 6 Plots of $(\alpha \mathrm{E})^{0.5}$ versus $\mathrm{E}$ (a) and $(\alpha \mathrm{E})^{2}$ versus $\mathrm{E}(\mathrm{b})$ for pristine HPC and HPC/BT composite films

\subsection{Refractive index and optical dispersion parameters}

The refractive index (n) is an optical parameter that is connected to the light speed through the analyzed material. The experimentally determined $\mathrm{n}$ values of pristine HPC and HPC/BT composite films as a function of wavelength are displayed in Figure 7a. For all samples the refractive index is decreasing as the light wavelength is gradually increasing. Furthermore, incorporation of BT filler determines the increase of the refractive index comparatively to the pure HPC, namely, at $486 \mathrm{~nm}, n$ ranges from 1.533 at $0 \% \mathrm{BT}$ to 1.605 at $2 \% \mathrm{BT}$ in the matrix. This kind of enhancement in the refractive index magnitude might be the result of an increase of the composite density, as supported by literature data for polymer composites (Banerjee et al., 2019). Comparable results were reported for other polymer/BT composites, namely the refractive index of HPC/BT samples is smaller than that of poly(arylene ether ketone)/BT (1.65 at $12 \mathrm{wt} \%$ BT) (Imai et al., 2010), similar to that of PMMA/BT (1.56 at 10 vol\% BT) (Nagao et al., 2011) and higher than that of PMMA/ethylene glycol monovinyl ether/BT (1.53 for $\mathrm{BaTiO}_{3} / 8 \mathrm{EGMVE} / 10 \mathrm{H}_{2} \mathrm{O} / 5 \mathrm{PMMA}$ ) (Mimura et al., 2010).

Optical dispersion parameters can be obtained by making a close analysis of the refractive index dependence on photon energy. This is described by the Wemple and DiDomenico (WDD) single oscillator model, as shown by equation (4):

$n^{2}=1+\frac{E_{d} E_{0}}{E_{0}^{2}-E^{2}}$

where $E_{0}$ is the average excitation energy for electronic transitions and $E_{d}$ is the dispersion energy.

Figure $7 b$ displays the plots of $1 /\left(n^{2}-1\right)$ against $E^{2}$ for HPC matrix and its BT-containing composites. The values of $\mathrm{E}_{0}$ and $\mathrm{E}_{\mathrm{d}}$ were estimated from the linear fit of the obtained graphs (Figure $7 b$ ) and the results are provided in Table 2. 
(a)

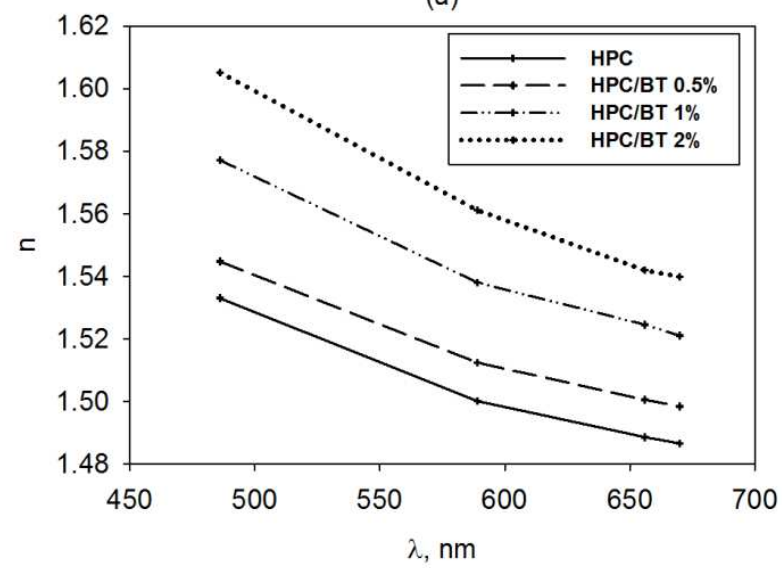

(b)

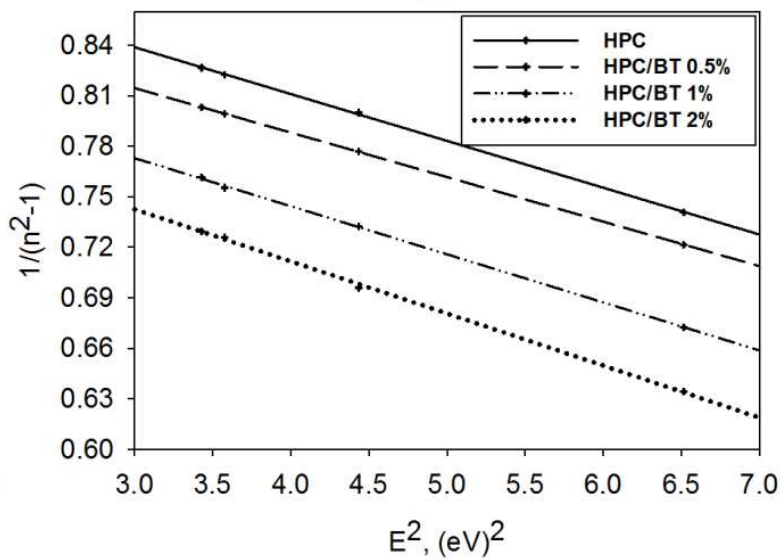

343

344

345

346

347

FIGURE 7 Refractive index dependence of wavelength (a) and plots of $1 / \mathrm{n}^{2}-1$ versus $\mathrm{E}^{2}$ (b) for neat HPC and HPC/BT composite films

The obtained data indicate that the strength of the interband optical transitions, expressed by $E_{\mathrm{d}}$, are decreasing as the filling level is higher. The average excitation energy $\mathrm{E}_{0}$ follows the same trend. This behavior was also noticed for polyvinyl alcohol (PVA) filled with BT (Barzic et al., 2020). The oscillator energy varies analogously to the optical band gap energy as the reinforcement level in HPC is higher. However, the values of $E_{d}, E_{0}$ and $n_{0}$ for HPC/BT are slightly smaller than those reported for PVA/BT (Barzic et al., 2020).

TABLE 2 The values of dispersion energy $\left(E_{d}\right)$, single-oscillator energy $\left(E_{0}\right)$, static refractive index $\left(\mathrm{n}_{0}\right)$, linear optical susceptibility $\left(\chi^{(1)}\right)$, third order optical susceptibility $\left(\chi^{(3)}\right)$, and nonlinear refractive index $\left(\mathrm{n}_{\mathrm{NL}}\right)$ for HPC and $\mathrm{HPC} / \mathrm{BT}$ composite films

\begin{tabular}{|l|c|c|c|c|c|c|}
\hline Sample code & $\mathrm{E}_{\mathrm{d}}(\mathrm{eV})$ & $\mathrm{E}_{0}(\mathrm{eV})$ & $\mathrm{n}_{0}$ & $\chi^{(1)}$ & $\chi^{(3)}($ e.s.u. $)$ & $\mathrm{n}_{\mathrm{NL}}(\mathrm{e} . \mathrm{s.u})$. \\
\hline HPC & 6.900 & 6.363 & 1.444 & 0.086 & $9.43 \cdot 10^{-15}$ & $2.46 \cdot 10^{-13}$ \\
\hline HPC/BT 0.5\% & 6.510 & 5.818 & 1.456 & 0.089 & $1.07 \cdot 10^{-14}$ & $2.77 \cdot 10^{-13}$ \\
\hline HPC/BT 1\% & 6.393 & 5.488 & 1.471 & 0.093 & $1.26 \cdot 10^{-14}$ & $3.23 \cdot 10^{-13}$ \\
\hline HPC/BT 2\% & 6.225 & 5.199 & 1.482 & 0.095 & $1.40 \cdot 10^{-14}$ & $3.56 \cdot 10^{-13}$ \\
\hline
\end{tabular}

The data concerning $E_{0}$ and $E_{d}$ can be utilized to calculate the static refractive index $\left(n_{0}\right)$ at zero photon energy, as revealed by equation (5):

$n_{0}=\left(1+\frac{E_{d}}{E_{0}}\right) 0.5$

As seen in Table 2, the static refractive index is higher as the inorganic particle amount in HPC matrix is increasing. 
Optical dispersion parameters lie at the basis for determining nonlinear optical properties (Soliman et al., 2020), namely optical susceptibility and nonlinear refractive index, defined by equations (68):

$\chi^{(1)}=\frac{E_{d} / E_{0}}{4 \pi}$

$\chi^{(3)}=6.82 \cdot 10^{-15} \cdot\left(E_{d} / E_{o}\right)^{4}$

$n_{N L}=\frac{12 \pi \chi^{(3)}}{n_{0}}$

where $\chi^{(1)}$ is the first-order optical susceptibility, $\chi^{(3)}$ is the third-order optical susceptibility and $\mathrm{n}_{\mathrm{NL}}$ is the nonlinear refractive index.

The achieved data for nonlinear optical properties are listed in Table 2. It was found that the insertion of ceramic particles in HPC generates the enhancement of the first and third order optical susceptibility and also of the nonlinear refractive index. The values of $\chi^{(1)}$ and $\chi^{(3)}$ for HPC/BT are smaller comparatively to those of PVA/BT (Barzic et al., 2020), while the nonlinear refractive index of studied samples is higher. These aspects sustain the suitability of the HPC/BT materials for nonlinear optical and photonic devices.

\subsection{Optical and electrical conductivities}

The optical conductivity is connected to the refractive index and the optical absorption coefficient at specific wavelengths. For the analyzed composite materials this parameter was evaluated using equation (9):

$$
\sigma_{\text {opt }}=\alpha \cdot n \cdot c / 4 \pi
$$

where $\sigma_{o p t}$ is the optical conductivity and $c$ is the speed of light in empty space.

The conductivity at optical frequencies is depicting the material's optical response caused by the travelling of the charge carrier under the action of external electromagnetic waves. Figure 8 illustrates the dependence of $\sigma_{o p t}$ with photon energy for the prepared samples. The optical conductivity of the pristine HPC film is not changing very much within energy interval of 1.85-2.55 eV. A similar behavior is noticed for the HPC composites containing $0.5-2 \%$ BT filler. At $2.1 \mathrm{eV}$, the optical conductivity increases with an order of magnitude from $10^{8}$ for HPC to $10^{9}$ for HPC/BT $2 \%$, which is smaller than that of PVA/BT (Barzic et al., 2020). The enhancement of optical conductivity could be attributed to the formation of new states within the band gap that enable 
electron crossing between the valence to the nearest state, as observed in the lowering of optical band gap (Table 1).

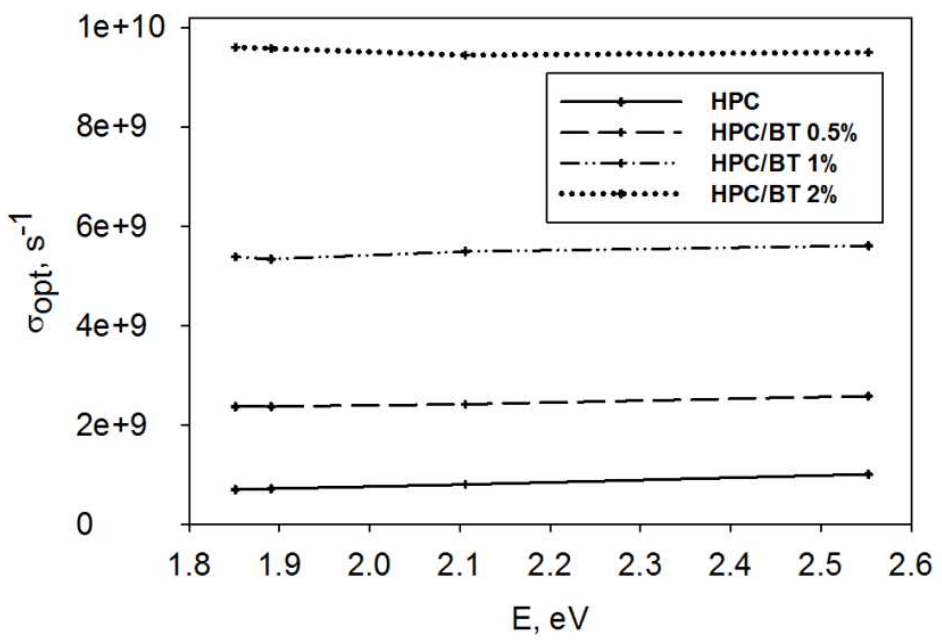

FIGURE 8 Optical conductivity versus photon energy for pristine HPC and HPC/BT composite films

The electrical conduction properties of HPC and HPC/BT composites were also analyzed at low frequencies. As seen in Figure 9, the measured conductivity $(\sigma)$ of all examined films is affected by the applied electric field frequency so that it ranges from $10^{-11} \mathrm{~S} / \mathrm{cm}$ to $10^{-7} \mathrm{~S} / \mathrm{cm}$. The conductivity ascended with the increase of frequency. A closer analysis shows that in the lower frequency interval of $1-10^{3} \mathrm{~Hz}$, the variation of $\sigma$ was gradual, whereas after $10^{3} \mathrm{~Hz}$ it ranged faster with further augmentation of the frequency. The recorded conductivity and the phase angle $(\theta)$ versus frequency are represented in Figures $9 \mathrm{a}$ and $\mathrm{b}$. The plateau region of conductivity retrieved at low frequencies connected with the values close to $0^{\circ}$ of phase angles are assigned with the DC conductivity (e.g. transport of charge carriers through the polymer matrix). On the other hand, the linear increase of conductivity at high frequencies and phase angle values close to $-90^{\circ}$ are generally specific to AC conductivity. Such behavior can be explained on the basis of Dyre's random free energy barrier approach (Dyre, 1988). The latter implies that conduction in solids occurs by bouncing of the charge carriers in the localized and highest states in the conduction band. This phenomenon is hasten by applying higher frequencies (Hassan et al., 2018; Tao et al., 2020).

On the other hand, the conductivity of HPC material is affected by the addition of ceramic filler. The increase in conduction features is more obvious up to $10^{3} \mathrm{~Hz}$ in the plateau region of conductivity, whereas after this point the slope of the conductivity curve is slightly increasing with addition of BT particles in the polymer. In other words, the conductivity increase in high frequency zone takes place owing to higher amount of free charges as a result of more polymer-nanoparticle 
interactions, combined with the existence of BT inside the polymer, which is known to promote the charge conduction (Morsi et al., 2019). Relatively constant conductivity in low frequency range might be ascribed to polar functional groups from HPC matrix, while for composites, this plateau results from electronic or ionic conductivity. As the filler amount in the system increases, the recorded conductivity increase could be attributed to carrier hopping among the macromolecular chains (Mendes et al., 2012). The level of conductivity at the highest frequency slightly exceeds that reported by Morsi et al for BT incorporated in PEO/CMC at low frequencies (Morsi et al., 2019).

(a)

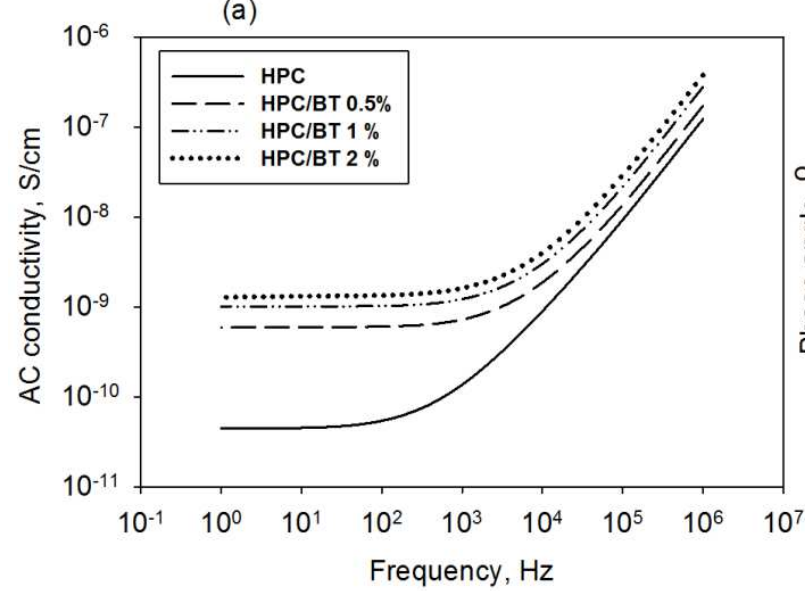

(b)

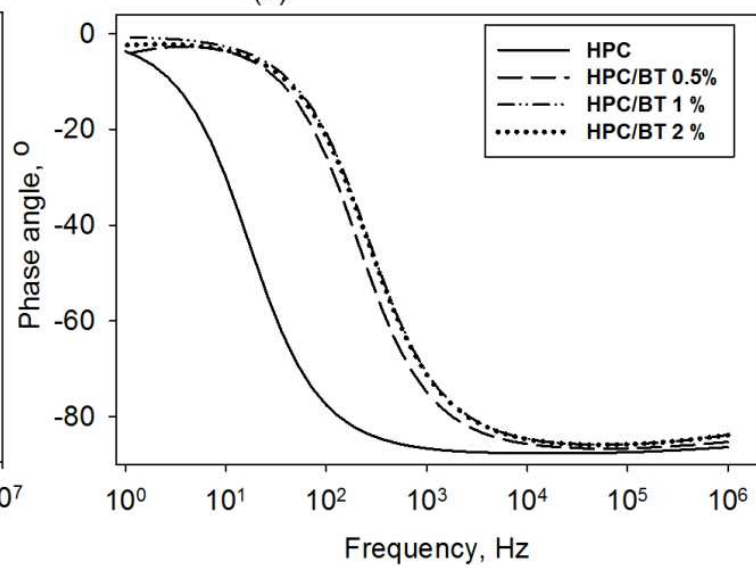

FIGURE 9 Measured conductivity versus frequency for neat HPC and HPC/BT composites

\subsection{Dielectric constant and electric energy density}

Dielectric spectroscopy was also used to test the dielectric performance of the samples. Figures 10a and $b$ illustrate the double-logarithmic dependence of the real $\left(\varepsilon^{\prime}\right)$ and imaginary $\left(\varepsilon^{\prime \prime}\right)$ dielectric constant on frequency (f) for neat HPC and HPC/BT composite films. The prepared film of HPC matrix has a permittivity of 3.4-5.2 in the recorded frequency domain. This result is in agreement with that reported by Abdelaziz (Abdelaziz, 2015). However, the obtained $\varepsilon^{\prime}$ magnitude for HPC is slightly smaller than that measured by Shinouda et al ( $\left.\varepsilon^{\prime} \sim 7.6\right)$ (Shinouda \& Abdel Moteleb, 2005) or by Maximean et al ( $\varepsilon^{\prime} \sim 19.9$ ) (Manaila Maximean et al., 2018). The differences could reside from the degree of sample drying, synthesis conditions (influencing the degree of substitution) or polymer processing (film, powder, fiber). It is evident that $\varepsilon^{\prime}$ lowers with increasing frequency over the interval of $1-10^{6} \mathrm{~Hz}$. The intensive decline of $\varepsilon^{\prime}$ observed at low frequencies for HPC/BT composite samples is specific to the electrode polarization (EP) process (Samet et al., 2015). This effect may appear due to the agglomeration of mobile charges mainly originated from BT source of charge carriers at the contact between sample and the electrodes used for dielectric measurements. 
As frequency increases, the EP effect is gradually diminished and a slighter reduction of $\varepsilon$ ' is noticed. The latter behavior reveals the 'pure' dipolar activity of the samples. If the frequency is very high, the dipoles can no longer cope-up with oscillations of the electric field, so the macromolecules are not orienting along the direction of the applied field (Abdelaziz \& Ghannam, 2010). For this reason, as the frequency increases, the dielectric constant gradually becomes smaller and is almost constant at high frequencies.

Generally, for reinforced polymers, the dielectric properties are linked to the filler content and particle distribution (Morsi et al., 2019). As one may see in Figure 10a, the real part of the dielectric constant is enhanced upon BT addition within HPC matrix. The permittivity of HPC/BT below 10 $\mathrm{Hz}$ were noticed to be high. At $1 \mathrm{kHz}$, the measured dielectric constant ranges from 4.2 for neat HPC up to 8.5 for HPC/BT 2\% (see the inset graph from Figure 10a).

As observed in Figure 10b, the dielectric loss is also higher with further addition of BT particles. This increase reveals that the charge carrying capacity of the prepared samples is notably larger with respect to unfilled HPC film. In addition, the high value of dielectric loss noted at low frequency (under $1 \mathrm{kHz}$ ) could be attributable to the mobile charges from the cellulosic matrix. The linear decrease of $\varepsilon$ " retrieved at low frequencies is connected with the $\sigma_{\mathrm{DC}}$ plateau region from Figure 9a and may be attributed to mobile charge carriers from the material.
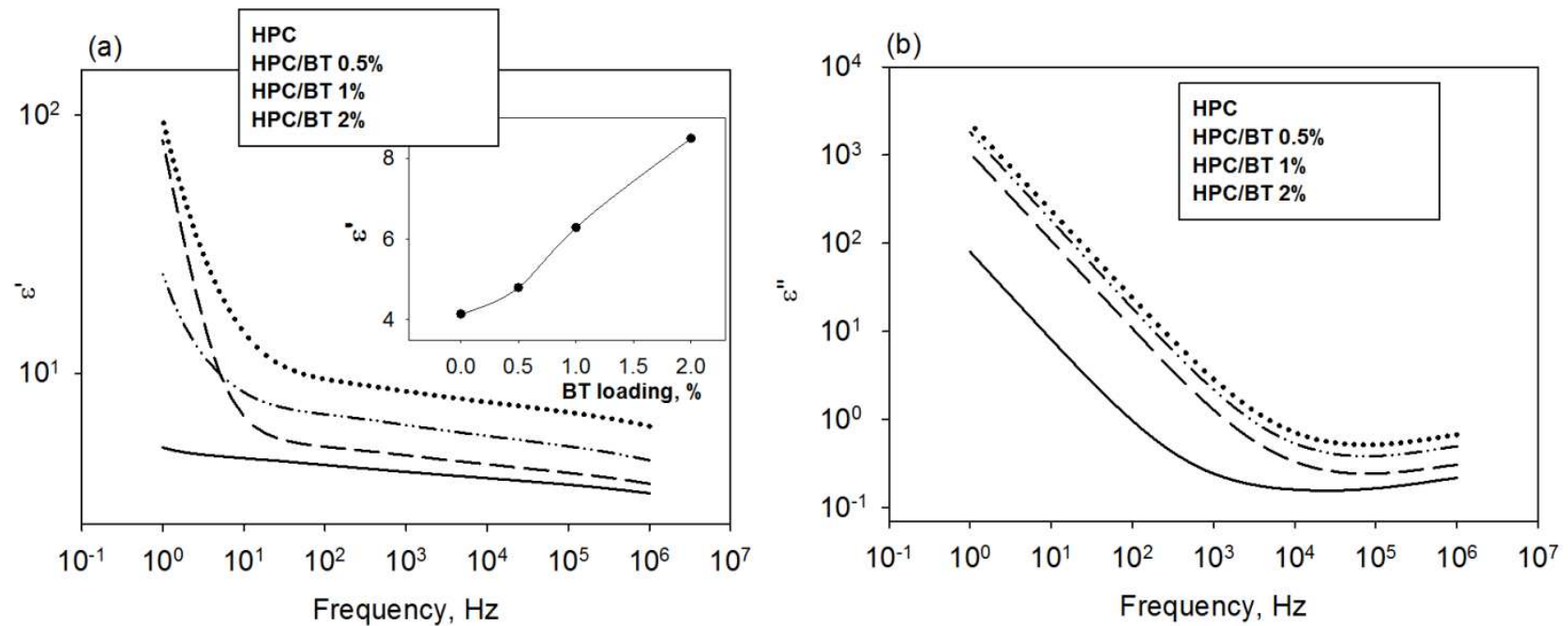

FIGURE 10 Real (a) and imaginary (b) dielectric constant against frequency for unfilled HPC and HPC/BT composite films. Inset graph shows variation of real part of permittivity at $1 \mathrm{kHz}$ with BT loading

On the other hand, the low signal from high frequencies is connected with the linear increase of conductivity and attributed to the loss of energy due to polarization of chemical dipoles. Similar dielectric behavior was reported for other polymers filled with BT ceramic material (Yao et al., 
2018; Ahmed et al., 2018; Beena \& Jayanna, 2019). However, at $1 \mathrm{kHz}$ and 2\% BT in HPC, the prepared composites have slightly smaller values for $\varepsilon^{\prime}$ and $\varepsilon^{\prime \prime}$ than those found in literature for PVA/BT (Beena \& Jayanna, 2019), polypyrrole/BT (Ahmed et al., 2018), while the permittivity of our samples is higher than that reported for polypropylene/BT (Yao et al., 2018) and PEO/CMC/BT (Morsi et al., 2019).

Dielectric properties affect the level of energy density within the prepared materials. From practical point of view, this aspect is essential for energy storage applications. The electric energy density (U) for a dielectric is described by equation (10):

$$
U=\varepsilon_{0} \varepsilon_{r} E^{2}
$$

where $\varepsilon_{0}$ is permittivity of free space, $\varepsilon_{\mathrm{r}}$ is the relative permittivity and $\mathrm{E}$ is the intensity of the electric field.

It is obvious that the discharged energy density of a dielectric can be amended by enhancing either the permittivity of the material or the field intensity. A close analysis of equation (10) indicates that the effect of field's intensity has a greater impact on U magnitude because it appears in relation in the second order. The highest energy density is obtained at the point of material's breakdown strength. Reinforced polymers have received special attention owing to the possibility to control the electrical characteristics in regard to the properties of single phase materials (Huang \& Jiang, 2015), whilst having the opportunity for upgrading the mechanical properties, in terms of mechanical flexibility or toughness. Ferroelectric particles, such as BT, are ideal for improving the dielectric performance of the polymers owing to their high permittivity.

According to Stark and Garton model (Stark \& Garton, 1955), the dielectric breakdown strength ranges proportionally with yields stress and is the reverse of the materials permittivity. The yield stress for HPC was reported to be 3.5 MPa (Borges et al., 2004). Based on these aspects, for HPC matrix the breakdown strength is $4.367 \cdot 10^{8} \mathrm{~V} / \mathrm{m}$. Literature shows that addition of BT particles in a polymer determines lowering of the breakdown strength (Dou et al., 2009; Yu et al., 2013). Knowing that the dielectric breakdown of BT is $38 \cdot 10^{5} \mathrm{~V} / \mathrm{m}$ (Branwood et al., 1962), the corresponding values for the studied composites were estimated and depicted in Figure 11. In order to determine the maximum values of the electric energy density, this parameter was assessed at electric field intensities corresponding to the breakdown strength. 


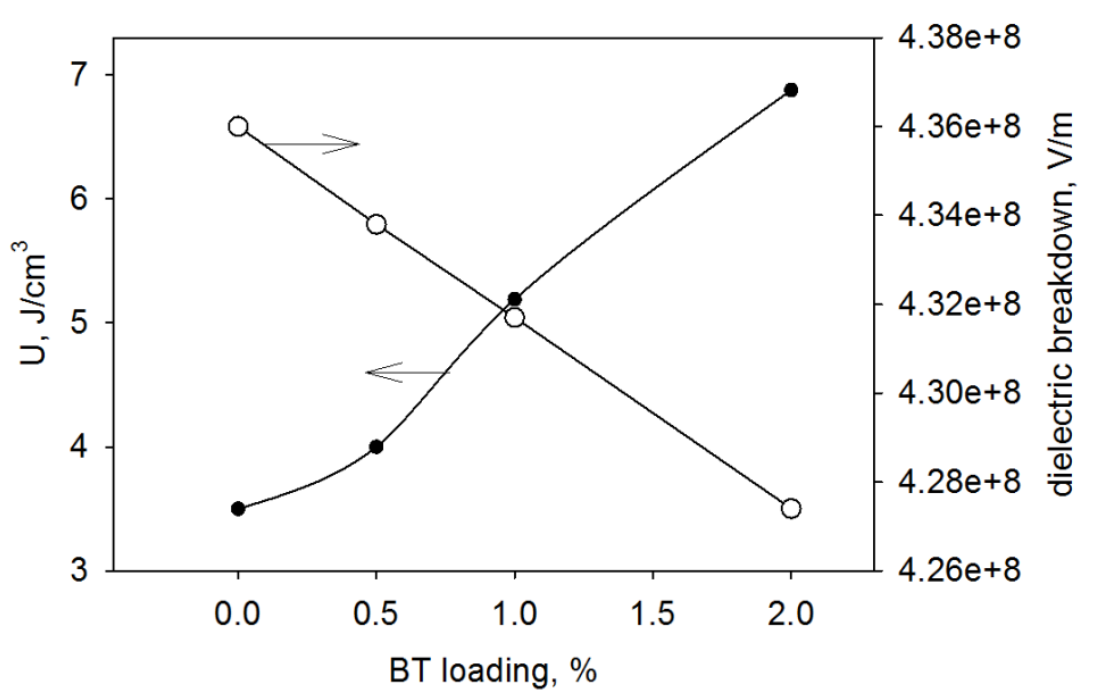

\section{CONCLUSIONS}

New polymer composites were fabricated by embedding ceramic particles in a cellulose derivative matrix. Addition of BT filler favor production of supplementary localized energy states in the forbidden energy zone, thus reducing transparency of films and direct band gap energy from 6.12 $\mathrm{eV}$ for neat HPC to $5.74 \mathrm{eV}$ for HPC/BT 2\%. Optical micrographs indicate a good dispersion of ceramic particles within HPC material owing to ultrasonication of the samples. Also, the presence of BT inclusions increases polarizability and implicitly the refractive index from 1.53 to 1.61 at 489 $\mathrm{nm}$. Moreover, optical and AC conductivity of HPC is improved after reinforcement, together with dielectric constant that increases from 4.2 for pristine HPC up to 8.5 for HPC/BT $2 \%$. This is reflected in a higher electric energy density and recommends the studied materials for energy storage applications.

FIGURE 11 Electric energy density for neat HPC and HPC/BT composites as a function of BT loading

Figure 11 shows the variation of electric energy density with BT amount introduced in HPC. Similar values to our results were reported for polyimide/reduced BT and poly(vinyl alcohol)/BT composites (Yue et al., 2019; Liu et al., 2020). The increase of U parameter with further addition of ceramic inclusions in the cellulose derivative matrix is a promising indicative for energy storage applications. 
Funding: Not applicable.

Conflicts of interest/Competing interests: The authors declare no conflict of interests.

Ethics approval: Not applicable.

Consent to participate: All authors consent to participate at the research included in this work and 530 approve the manuscript submission.

Consent for publication: All authors agree with article publication.

\section{REFERENCES}

Abdel-Halim, E. S., Alanazi, H. H., \& Al-Deyab, S. S. (2015). Utilization of olive tree branch cellulose in synthesis of hydroxypropyl carboxymethyl cellulose. Carbohydrate Polymers, 127, 124-134.

Abdelaziz, M., \& Ghannam, M. M. (2010). Influence of titanium chloride addition on the optical and dielectric properties of PVA films. Physica B: Condensed Matter, 405, 958-964. Abdelaziz, M. (2015). Effect of Sm3+ substitution on structural, optical and dielectric properties of hydroxypropyl cellulose films. Journal of Materials Science: Materials in Electronics, 26(11), 8688-8697. Ahmed, K., Kanwal, F., Ramay, S. M., Atiq, S., Rehman, R., Ali, S. M., \& Alzayed, N. S. (2018). Synthesis and Characterization of BaTiO3/Polypyrrole Composites with Exceptional Dielectric Behaviour. Polymers, 10, 1273.

550 Banerjee, M., Jain, A., \& Mukherjee, G. S. (2019). Microstructural and optical properties of 551 polyvinyl alcohol/manganese chloride composite film. Polymer Composites, 40, E765-E775.

552 Barzic, A. I., Soroceanu, M., Rotaru, R., Harabagiu, V., \& Ciobanu, R. C. (2020). Optical 553 Dispersion Characteristics of Polyvinyl Alcohol Reinforced with a Nanoceramic Filler. Materiale 554 Plastice (Mater. Plast.), 57, 1-7. 
Beena, P., \& Jayanna, H. S. (2019). Dielectric studies and AC conductivity of piezoelectric barium titanate ceramic polymer composites. Polymers and Polymer Composites, 27, 619-625.

Borges, J. P., Godinho, M. H., \& Martins, A. F. (2004). Tensile Properties of Cellulose Fiber Reinforced Hydroxypropylcellulose Films. Polymer Composites, 25, 102-110.

Branwood, A., Hughes, O. H., Hurd, J. D., \& Tredgold, R. H. (1962). Dielectric breakdown in barium titanate. Proc Phys Soc, 79, 1161.

Choudhary, S., \& Sengwa, R. J. (2018). ZnO nanoparticles dispersed PVA-PVP blend matrix based high performance flexible nanodielectrics for multifunctional microelectronic devices. Current Applied Physics, 18, 1041-1058.

Dou, X., Liu, X., Zhang, Y., Feng, H., Chen, J.-F., \& Du, S. (2009). Improved dielectric strength of barium titanate-polyvinylidene fluoride nanocomposite. Applied Physics Letters, 95(13), 132904.

Dyre, J. C. (1988). The random free-energy barrier model for ac conduction in disordered solids. Journal of Applied Physics, 64, 2456.

Gao, L., Liu, Q., Gao, Z., \& Lin, Y. (2008). Preparation and characterization of polyimide/silica-barium titanate nanocomposites. Polymer Composites, 29, 1160-1164.

Goyal, R. K., Katkade, S. S., \& Mule, D. M. (2013). Dielectric, mechanical and thermal properties of polymer/BaTiO3 composites for embedded capacitor. Composites Part B: Engineering, 44, 128132.

Gu, L., Li, T., Xu, Y., Sun, C., Yang, Z., Zhu, D., \& Chen, D. (2019). Effects of the particle size of $\mathrm{BaTiO} 3$ fillers on fabrication and dielectric properties of $\mathrm{BaTiO} 3 /$ polymer/Al films for capacitor energy-storage application. Materials, 12, 439.

Hassan, M. L., Ali, A. F., Salama, A. H., \& Abdel-Karim, A. M. (2018). Novel cellulose nanofibers/barium titanate nanoparticles nanocomposites and their electrical properties. Journal of Physical Organic Chemistry, 32, e3897.

Hua, Z., Shi, X., \& Chen, Y. (2019). Preparation, structure, and property of highly filled polyamide 11/BaTiO3 piezoelectric composites prepared through solid-state mechanochemical method. Polymer Composites, 40, E-177-E-185.

Huang X., \& Jiang P. (2015). Core-Shell Structured High-k Polymer Nanocomposites for Energy Storage and Dielectric Applications. Advanced Materials, 27, 546-554.

Huang, X., Zeng, J., Yu, H., Wei, X., Pu, L., \& Liu, J. (2020). NiNb2O6-BaTiO3/poly(arylene ether nitriles) composite film dielectrics with excellent flexibility and high permittivity for organic film capacitors. Polymer Composites, 41, 94-101. 
Imai, Y., Terahara, A., Hakuta, Y., Matsui, K., Hayashi, H., \& Ueno, N. (2010). Synthesis and characterization of high refractiveindex nanoparticle/poly(arylene ether ketone) nanocomposites. Polymer Journal, 42,179-184.

Joshi, G., Rana, V., Naithani, S., Varshney, V. K., Sharma, A., \& Rawata, J. S. (2019). Chemical modification of waste paper: An optimization towards hydroxypropyl cellulose synthesis. Carbohydrate Polymers, 223, 115082.

Kesavan, K., Rajendran, S., \& Mathew, C. M. (2015). Influence of Barium Titanate on poly(vinyl pyrrolidone)-based composite polymer blend electrolytes for lithium battery applications. Polymer Composites, 36, 302-311.

Kobayashi, Y., Kurosawa, A., Nagao, D., \& Konno, M. (2010). Fabrication of Barium Titanate Nanoparticles-EpoxyResin Composite Films and Their Dielectric Properties. Polymer Composites, 31, 1179-1183.

Liu, G., Li, Y, Guo, B., Tang, M., Li, Q., Dong, J., Yu, L., Yu, K., Yan, Y., Wang, D., Zhang, L., Zhang, H., He, Z., \& Jin, L. (2020). Ultrahigh dielectric breakdown strength and excellent energy storage performance in lead-free barium titanate-based relaxor ferroelectric ceramics via a combined strategy of composition modification, viscous, polymer processing, and liquid-phase sintering. Chemical Engineering Journal, 398, 125625.

Lombardi, M., Guerriero, A., Kortaberria, G., Mondragon, I., Sangermano, M., \& Montanaro, L. (2011). Effect of the ceramic filler features on the properties of photopolymerized BaTiO3-acrylic composites. Polymer Composites, 32, 1304-1312.

Malathi, M., Tamilarasan, K., \& Ganesan, V. (2015). Role of ceramic reinforcement in composite polymer electrolyte. Polymer Composites, 36, $42-46$.

Manaila Maximean, D., Danila, O., Ganea, C. P., \& Almeida, P. L. (2018). Filling in the voids of electrospun hydroxypropyl cellulose network: Dielectric investigations. The European Physical Journal Plus, 133, 159.

Mendes, S. F., Costa, C. M., Caparros, C., Sencadas, V., \& Lanceros-Méndez, S. (2012). Effect of filler size and concentration on the structure and properties of poly (vinylidene fluoride)/BaTiO3 nanocomposites. Journal of Materials Science, 47, 1378-1388.

Mimura, K., Hiramatsu, K., Moriya, M., Sakamoto, W., Kawado, S., Uesu, Y., \& Yogo, T. (2010). Optical properties of transparent barium titanate nanoparticle/polymer hybrid synthesized from metal alkoxides. Journal of Nanoparticle Research, 12, 1933-1943.

Mohd Ishak, N. A., Khalil, I., Abdullah, F. Z., \& Julkapli, N. M. (2020). A correlation on ultrasonication with nanocrystalline cellulose characteristics. Carbohydrate Polymers, 246, 116553. 
Morsi, M. A., Abdelaziz, M., Oraby, A. H., \& Mokhles, I. (2019). Structural, optical, thermal, and dielectric properties of polyethylene oxide/carboxymethyl cellulose blend filled with barium titanate. Journal of Physics and Chemistry of Solids, 125, 103-114.

Nagao, D., Kinoshita, T., Watanabe, A., \& Konno, M. (2011). Fabrication of highly refractive, transparent BaTiO3/poly(methyl methacrylate) composite films with high permittivities. Polymer International, 60, 1180-1184.

Nishio, Y., \& Takahashi, T. (1984). Morphological study of hydroxypropyl cellulose films prepared from thermotropic melt under shear. Journal of Macromolecular Science, Part B: Physics, 23(4-6), 483-495.

Olmos, D., Montero, F., Gonzalez-Gaitano, G., \& Gonzalez-Benito, J. (2013). Structure and morphology of composites based on polyvinylidene fluoride filled with $\mathrm{BaTiO} 3$ submicrometer particles: Effect of processing and filler content. Polymer Composites, 34, 2094-2104.

Pant, H. C., Patra, M. K., Verma, A., Vadera, S. R., \& Kumar, N. (2006). Study of the dielectric properties of barium titanate-polymer composites. Acta Materialia, 54, 3163-3169.

Petrovsky, V., Petrovsky, T., Kamlapurkar, S., \& Dogan, F. (2008). Dielectric Constant of Barium Titanate Powders Near Curie Temperature. Journal of the American Ceramic Society, 91, 3590.

Rotaru, R., Peptu, C., \& Harabagiu, V. (2016). Viscose-barium titanate composite for electromagnetic shielding. Cellulose Chemistry and Technology, 50 (5-6), 621-628.

Rotaru, R., Peptu, C., Samoila, P., \& Harabagiu, V. (2017). Preparation of ferroelectric barium titanate through an energy effective solid state ultrasound assisted method. Journal of the American Ceramic Society, 100, 4511-4518.

Samet, M., Levchenko, V., Boiteux, G., Seytre, G., Kallel, A., \& Serghei, A. (2015). Electrode polarization vs. Maxwell-Wagner-Sillars interfacial polarization in dielectric spectra of materials: Characteristic frequencies, and scaling laws. The Journal of Chemical Physics, 142, 194703.

Sen, F., Basturk, E., Karadoğan, B., Madakbaş, S., \& Kahraman, M. V. (2016). Effect of barium titanate on the thermal, morphology, surface, and mechanical properties of the thermoplastic polyurethane/barium titanate composites. Polymer-Plastics Technology and Engineering, 55, 13251331.

Sengwa, R. J., Choudhary, S., \& Dhatarwal, P. (2019). Nonlinear optical and dielectric properties of $\mathrm{TiO} 2$ nanoparticles incorporated PEO/PVP blend matrix based multifunctional polymer nanocomposites. Journal of Materials Science - Materials in Electronics, 30, 12275-13294.

Sharma, R., Varshney, V. K., Chauhan, G. S., Naithani, S., \& Soni P. L. (2009). Hydroxypropylation of Cellulose Isolated from Bamboo(Dendrocalamus strictus) with Respect to 
653

654

655

656

657

658

659

660

661

662

663

664

665

666

667

668

669

670

671

672

673

674

675

676

677

678

679

680

681

682

683

684

685

686

HydroxypropoxylContent and Rheological Behavior of the HydroxypropylCellulose. Journal of Applied Polymer Science, 113, 2450-2455.

Shinouda, H. G., \& Abdel Moteleb, M. M. (2005). Dielectric Spectroscopy and Relaxation Phenomena of Moistened and Dry Polysaccharides. Journal of Applied Polymer Science, 98, 571582.

Silva, S. M. C., Pinto, F. V., Antunes, F. E., Miguel, M. G., Sousa, J. J. S., \& Pais, A. A. C. C. (2008). Aggregation and gelation in hydroxypropylmethyl cellulose aqueous solutions. Journal of Colloid and Interface Science, 327, 333-340.

Soliman, T. S., Vshivkov, S. A., \& Elkalashy, Sh. I. (2020). Structural, linear and nonlinear optical properties of $\mathrm{Ni}$ nanoparticles - Polyvinyl alcohol nanocomposite films for optoelectronic applications Optical Materials. Optical Materials, 107, 110037.

Stark, K., \& Garton, C. (1955). Electric Strength of Irradiated Polythene, Nature, 176, 1225-1226.

Takenaka, T. (2013). Lead-Free Piezoelectric Ceramics. In Handbook of Advanced Ceramics (Second Edition), Materials, Applications, Processing, and Properties (pp. 429-446). Academic Press.

Tao, J., Cao, S.-an, Feng, R., \& Deng, Y. (2020). High dielectric thin films based on barium titanate and cellulose nanofibrils. RSC Advances, 10, 5758-5765.

Tauc, J. (1967). The optical properties of solids. Physics Today, 20, 105.

Valiyaneerilakkal, U., Singh, A., Subash, C. K., Singh, K., Abbas, S. M., \& Varghese, S. (2015).

Preparation and characterization of poly(vinylidene fluoride-trifluoroethylene)/barium titanate polymer nanocomposite for ferroelectric applications. Polymer Composites, 38, 1655-1661.

Vanderghem, C., Jacquet, N., Danthine, S., Blecker, C., \& Paquot, M. (2012). Effect of physicochemical characteristics of cellulosic substrates on enzymatic hydrolysis by means of a multi-stage process for cellobiose production. Applied Biochemistry and Biotechnology, 166, 14231432.

Xu, W., Ding, Y., Jiang, S., Ye, W., Liao, X., \& Hou, H. (2016). High permittivity nanocomposites fabricated from electrospun polyimide/BaTiO3 hybrid nanofibers. Polymer Composites, 37, 794801.

Yang, Y., Pan, H., Xie, G., Jiang, Y., Chen, C., Su, Y., Wang, Y., \& Tai, H. (2020). Flexible piezoelectric pressure sensor based on polydopamine-modified BaTiO3/PVDF composite film for human motion monitoring. Sensors and Actuators A: Physical, 301, 111789.

Yao, J., Hu, L., Zhou, M., You, F., Jiang, X., Gao, L., Wang, Q., Sun, Z., \& Wang J. (2018). Synergistic Enhancement of Thermal Conductivity and Dielectric Properties in A12O3/BaTiO3/PP Composites. Materials, 11(9), 1536. 
687 Yogamalar, N. R., Kalpana, S., Senthil, V., \& Chithambararaj, A. (2018). In Multifunctional 688 Photocatalytic Materials for Energy (Eds.). Ferroelectrics for photocatalysis (pp. 307-324). 689 Woodhead Publishing.

690 Yu, K., Niu, Y., Xiang, F., Zhou, Y., Bai, Y., \& Wang, H. (2013). Enhanced electric breakdown 691 strength and high energy density of barium titanate filled polymer nanocomposites. Journal of 692 Applied Physics, 114, 174107.

693 Yue, S., Wan, B., Liu, Y., \& Zhang, Q. (2019). Significantly enhanced dielectric constant and 694 energy storage properties in polyimide/reduced BaTiO3 composite films with excellent thermal 695 stability. RSC Advances, 9, 7706-7717.

696 Zhang, C., Yin, Y., Yang, Q., Shi, Z., Hu, G. H., \& Xiong C. (2019). Flexible Cellulose/BaTiO3 697 Nanocomposites with High Energy Density for Film Dielectric Capacitor. ACS Sustainable 698 Chemistry \& Engineering, 7, 10641-10648. 
Figures
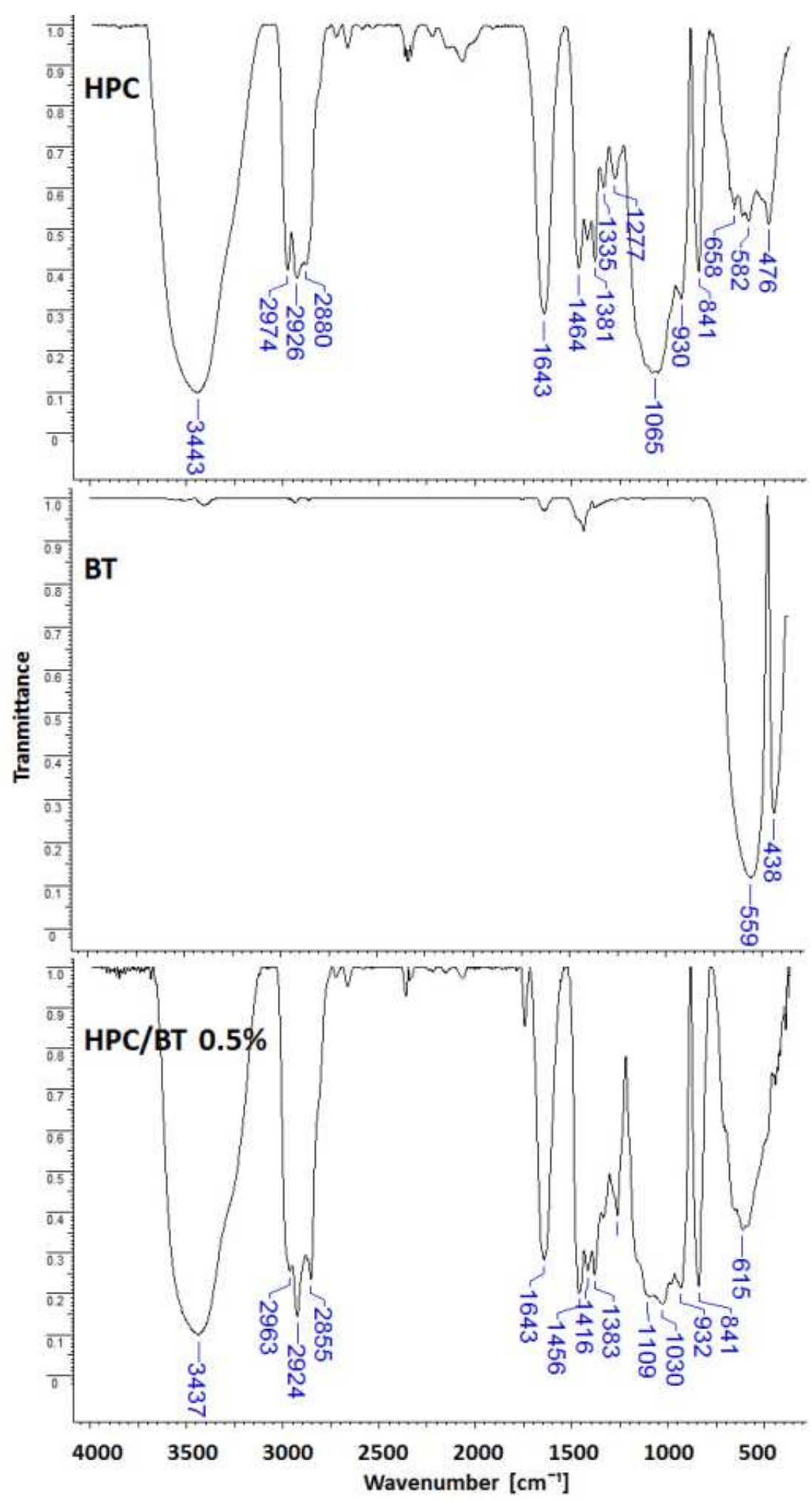

Figure 1

FTIR spectra of HPC film, BT powder, and HPC/BT 05\% composite films 

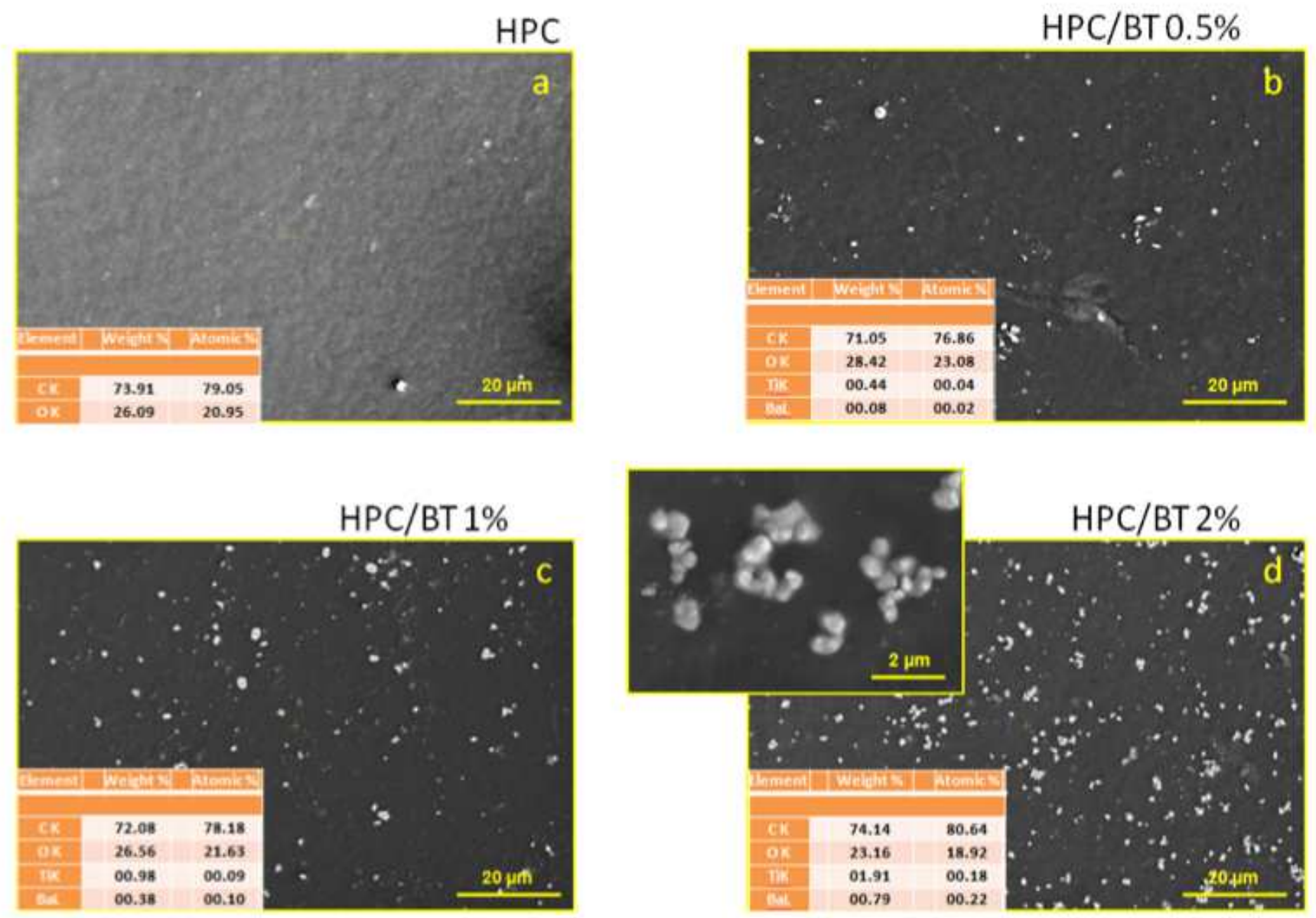

Figure 2

SEM micrographs of the surface of HPC and HPC/BT composites: (a) HPC, (b) HPC/BT 0.5\%, (c) HPC/BT $1 \%$ and (d) HPC/BT $2 \%$ 
HPC

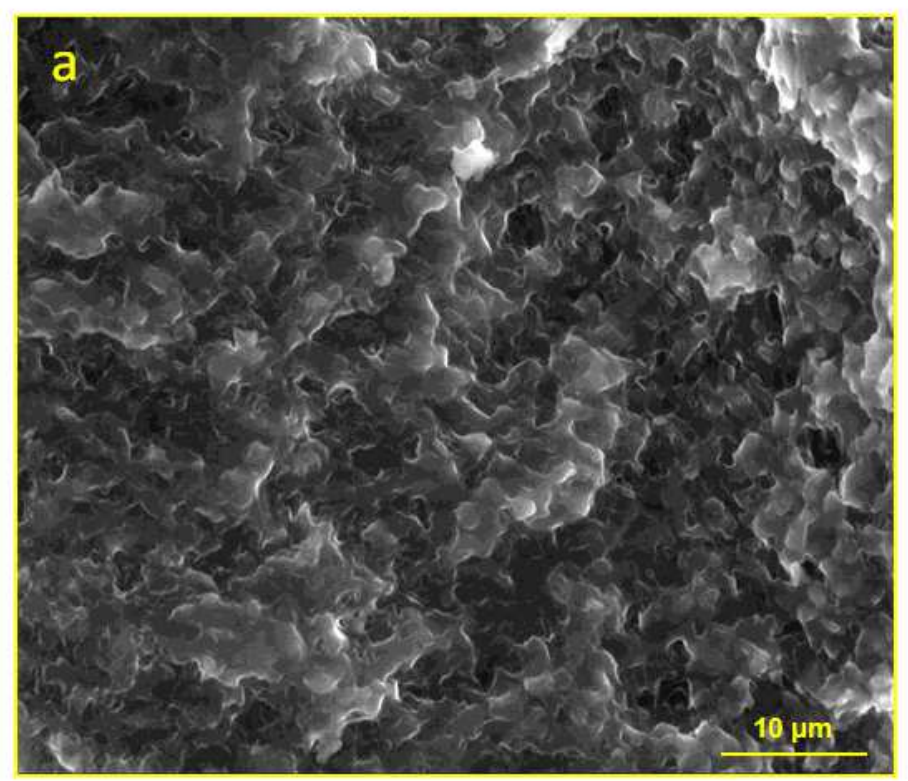

HPC/BT $1 \%$

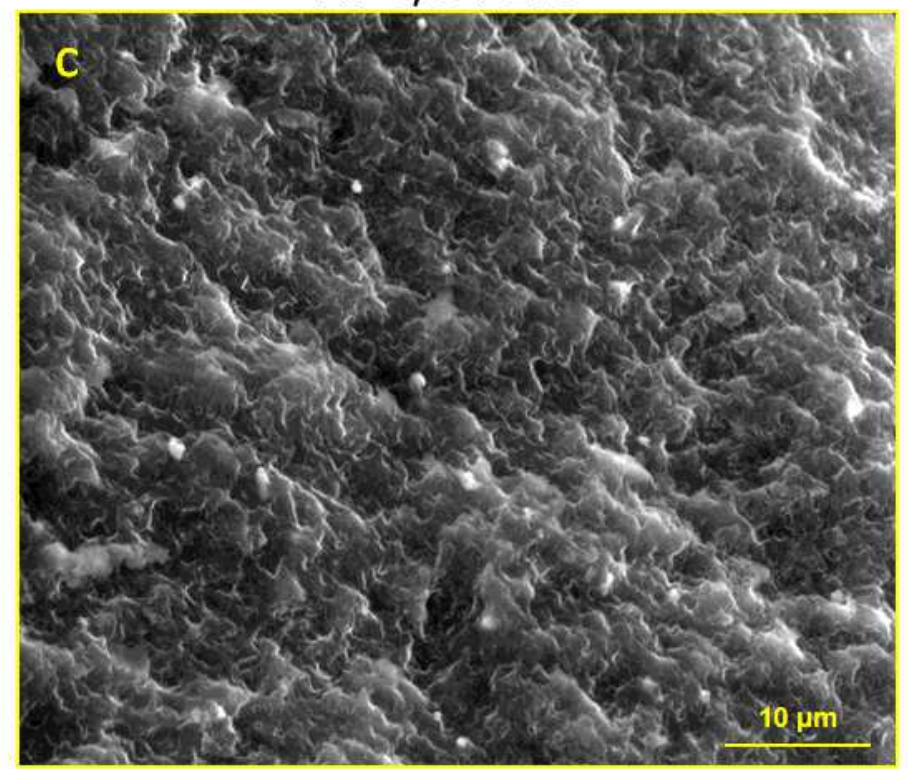

HPC/BT 0.5\%

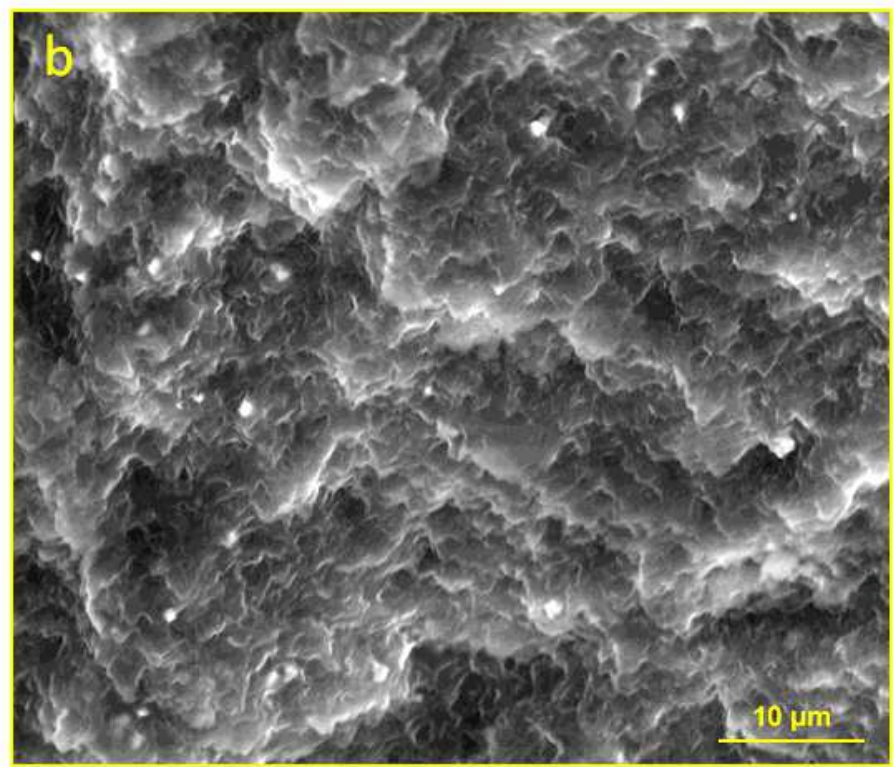

$\mathrm{HPC} / \mathrm{BT} 2 \%$

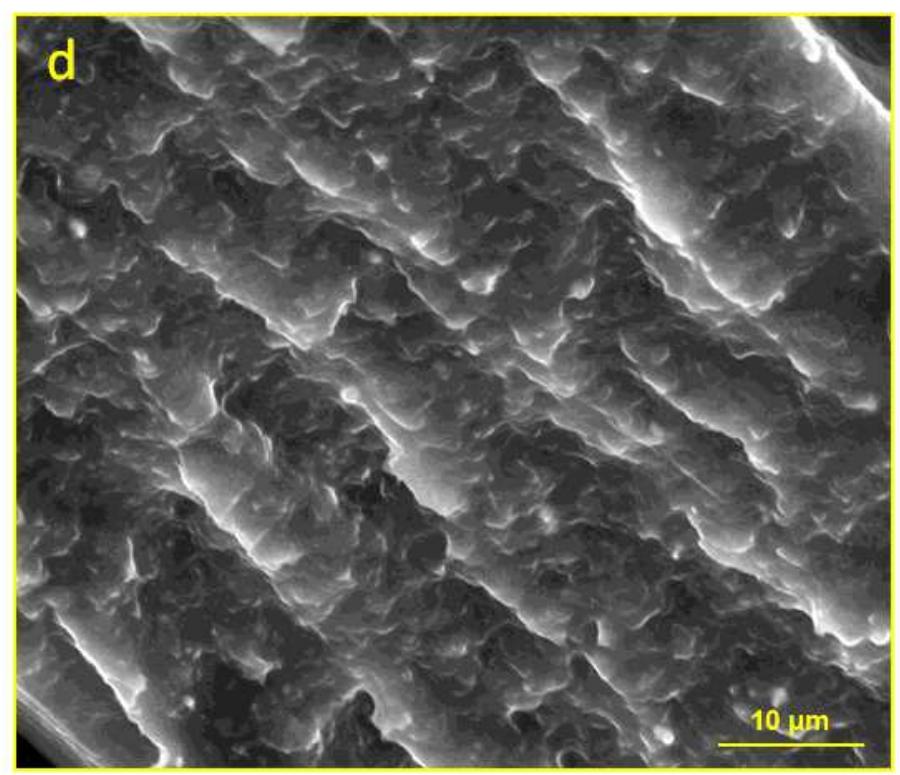

\section{Figure 3}

SEM micrographs: Cross-sections of HPC and HPC/BT composites films: (a) HPC, (b) HPC/BT 0.5\%, (c) HPC/BT $1 \%$ and (d) HPC/BT $2 \%$ 
(a)

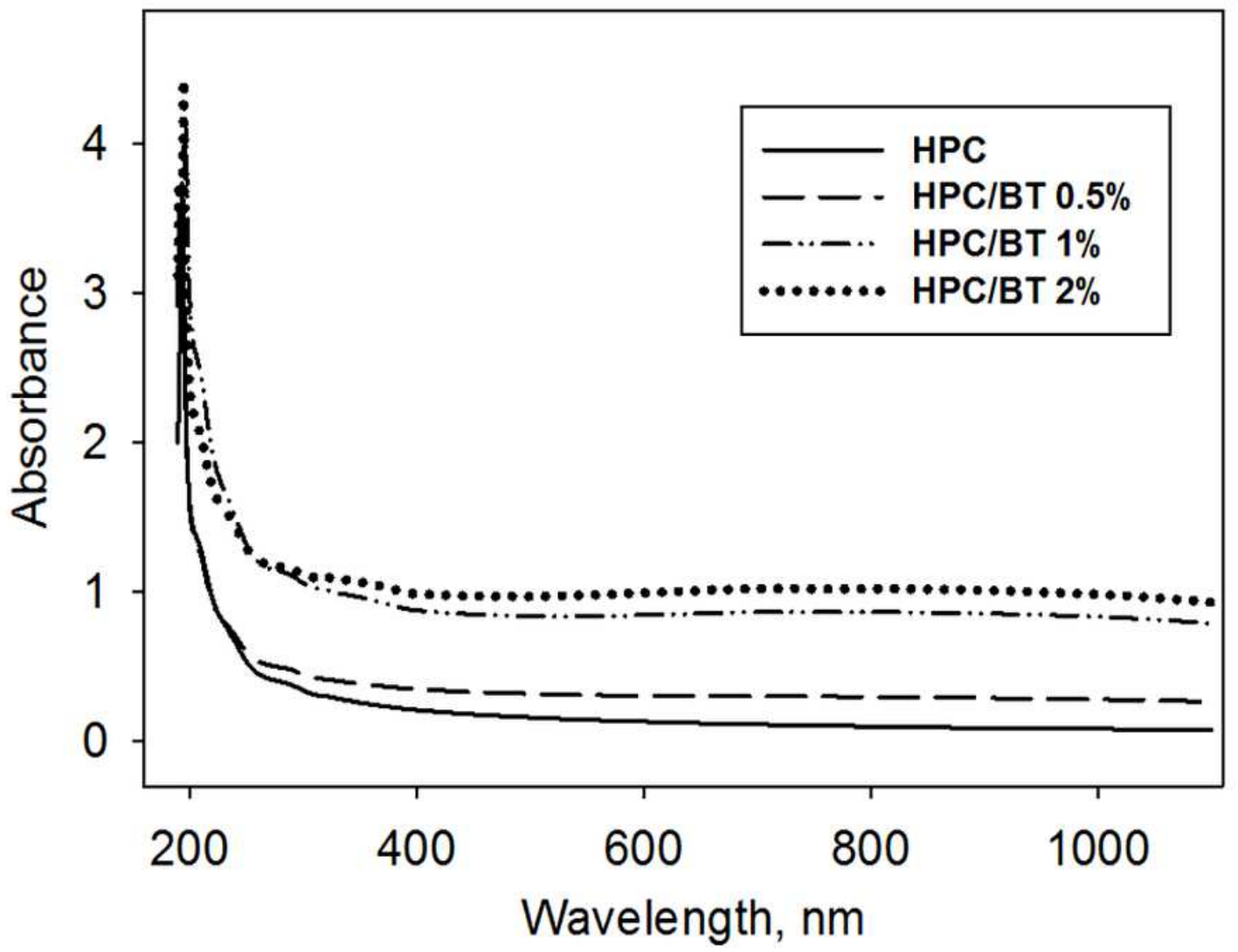

(b)
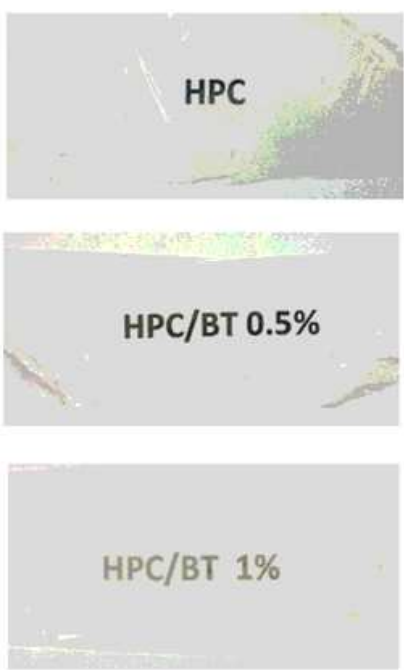

HPC/BT $2 \%$

Figure 4

UV-VIS-NIR spectrum of the neat HPC and HPC/BT composite films (a) and the pictures of analyzed samples (b) 


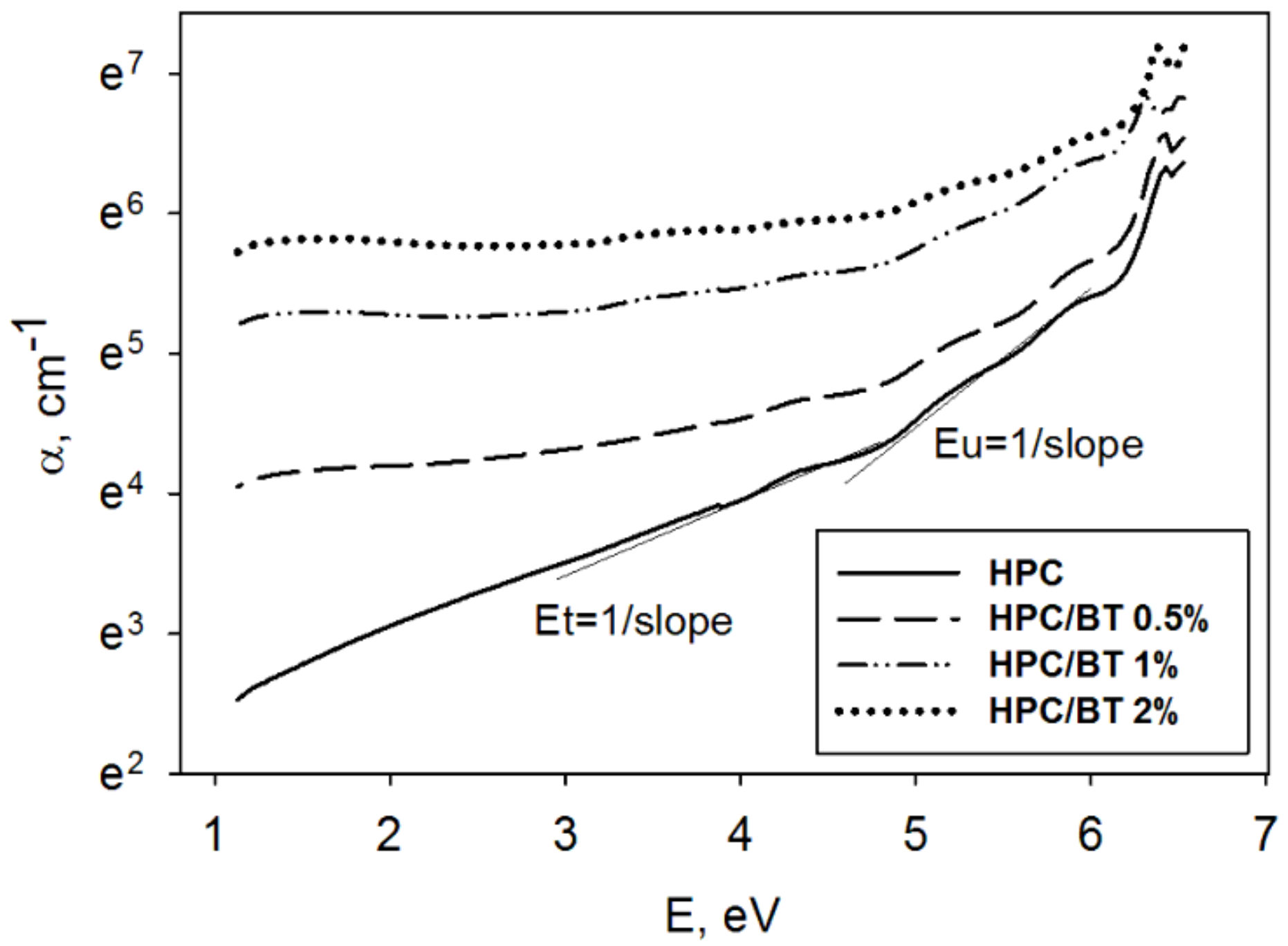

Figure 5

Optical absorption coefficient versus photon energy for unfilled HPC and HPC/BT composite films 
(a)

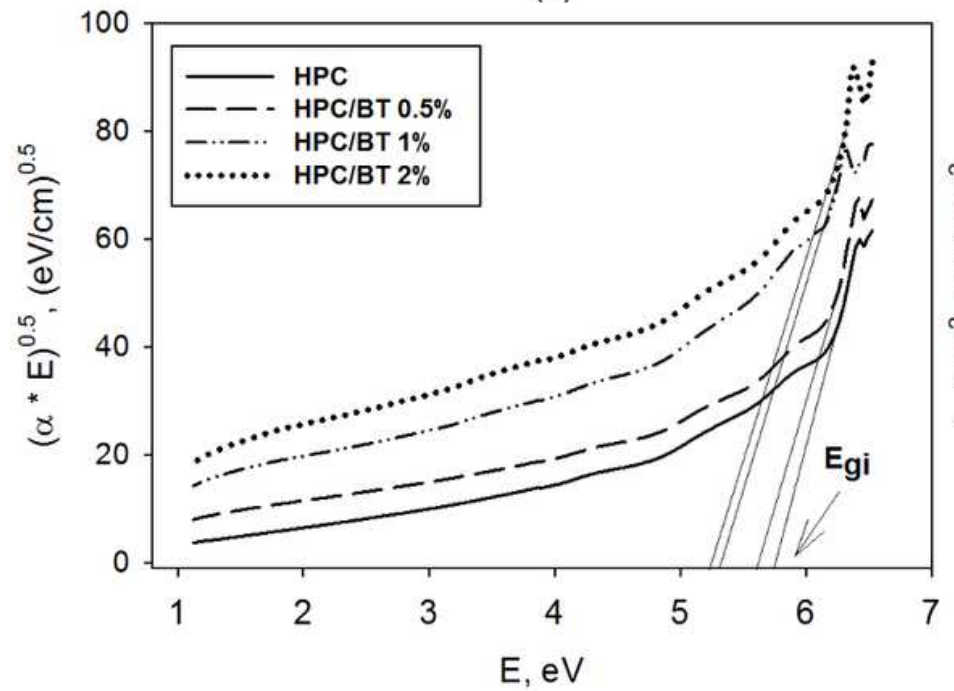

(b)

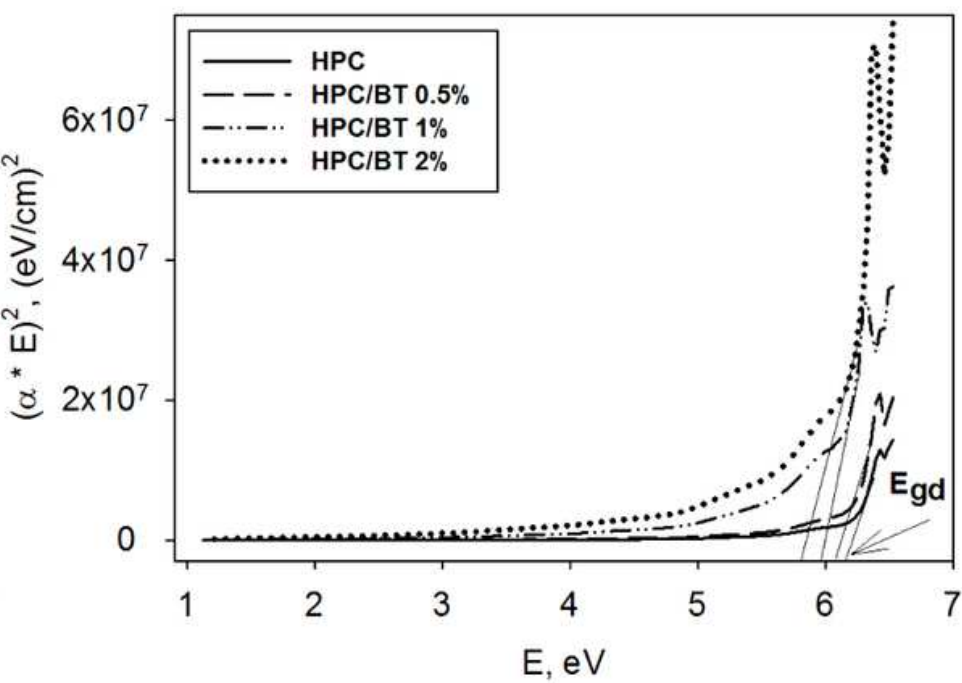

Figure 6

Plots of (aE) 0.5 versus $E(a)$ and $(a E) 2$ versus $E(b)$ for pristine HPC and HPC/BT composite films

(a)

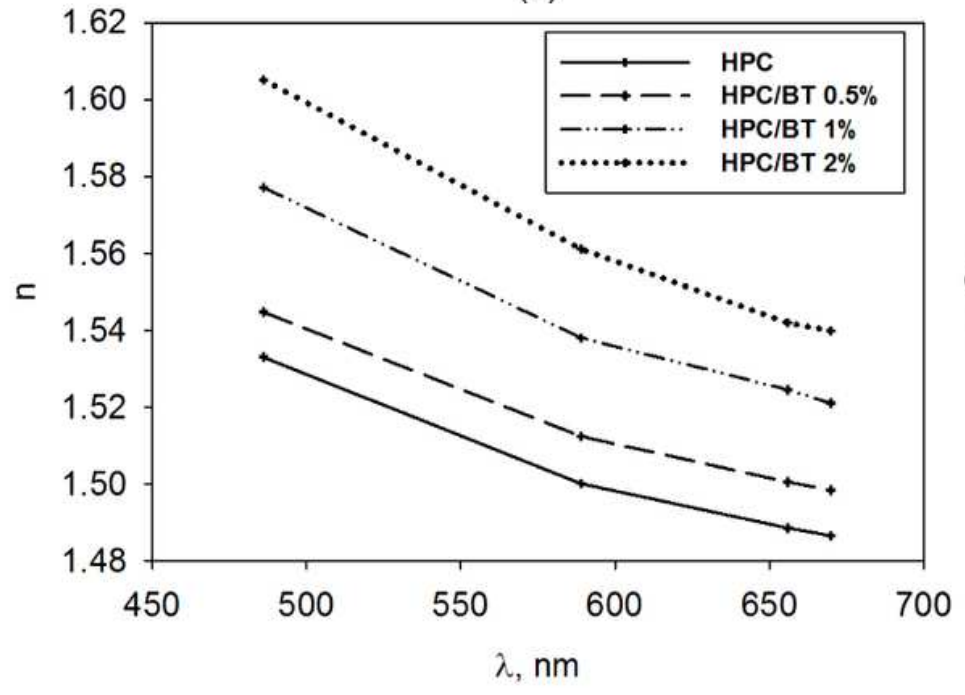

(b)

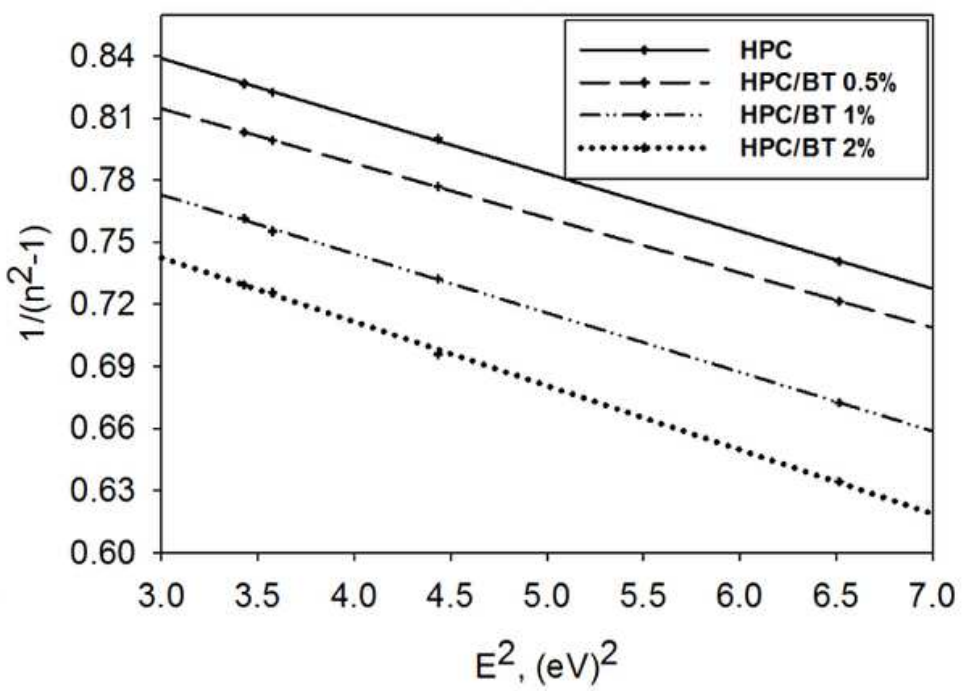

Figure 7

Refractive index dependence of wavelength (a) and plots of 1/n2-1 versus E2 (b) for neat HPC and HPC/BT composite films 


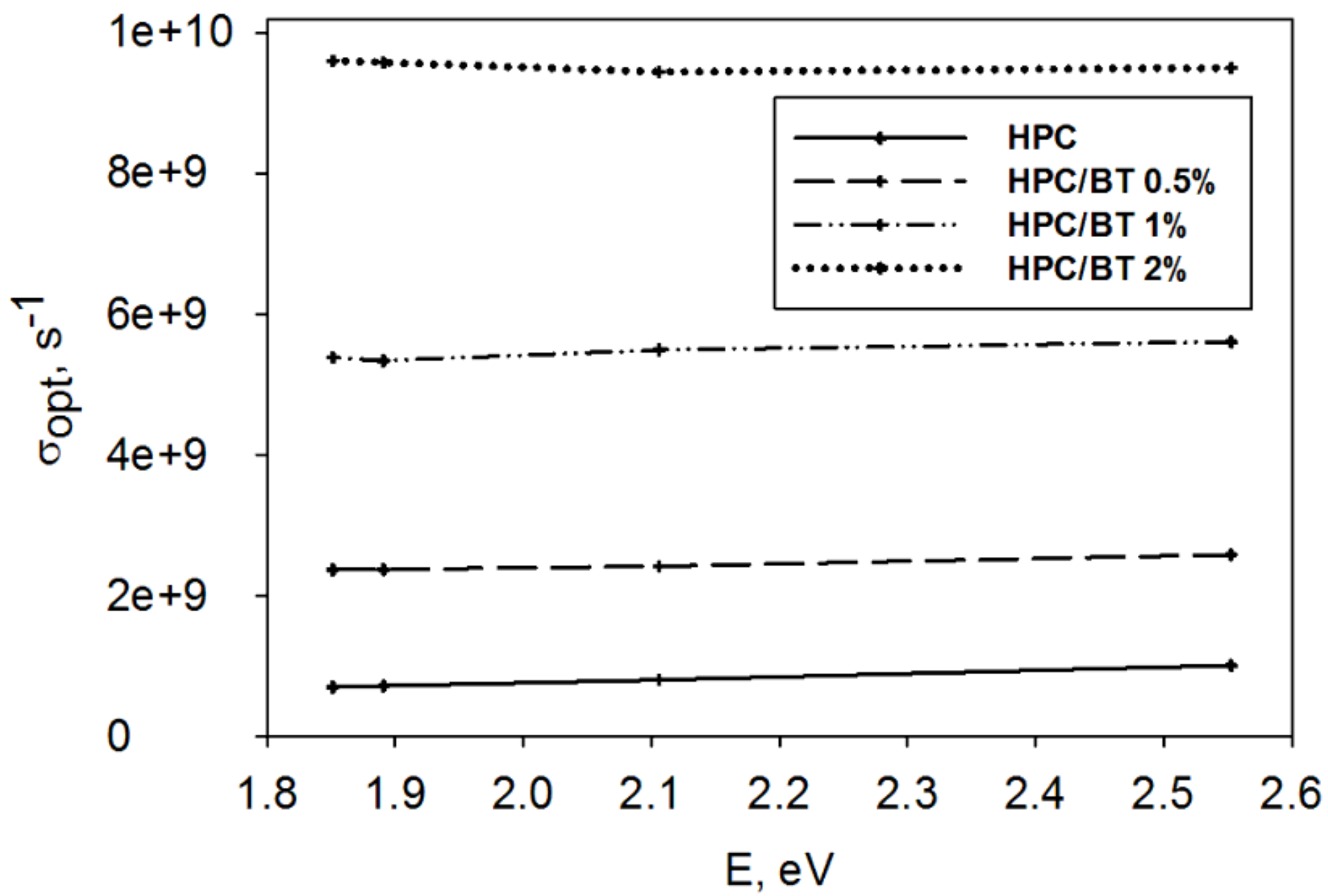

Figure 8

Optical conductivity versus photon energy for pristine HPC and HPC/BT composite films
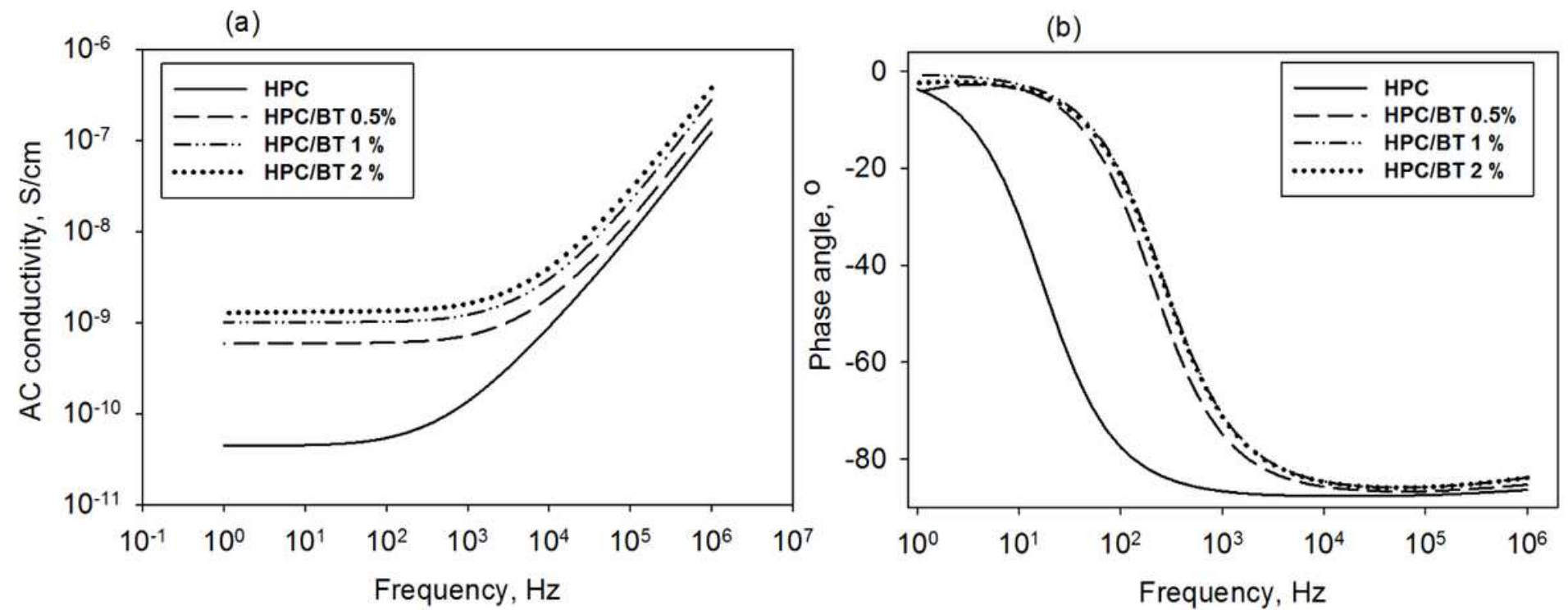
Figure 9

Measured conductivity versus frequency for neat HPC and HPC/BT composites
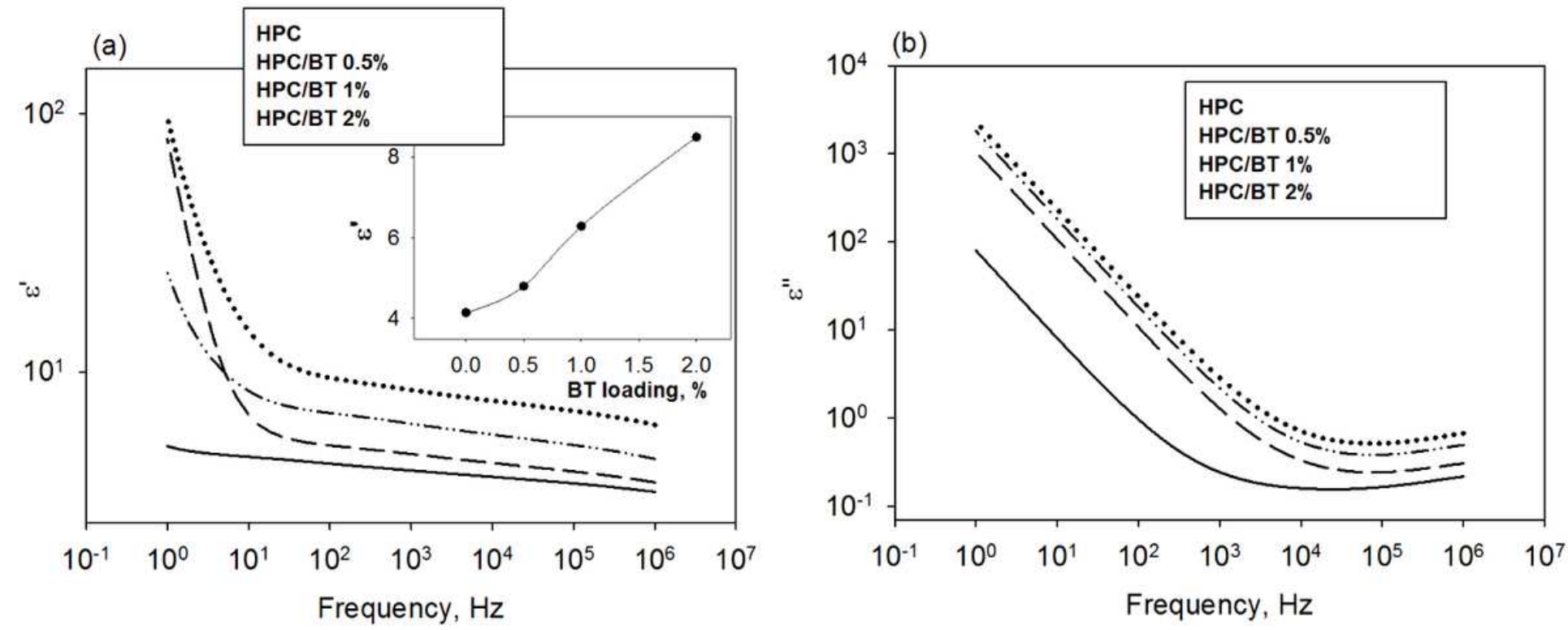

Figure 10

Real (a) and imaginary (b) dielectric constant against frequency for unfilled HPC and HPC/BT composite films. Inset graph shows variation of real part of permittivity at $1 \mathrm{kHz}$ with BT loading 


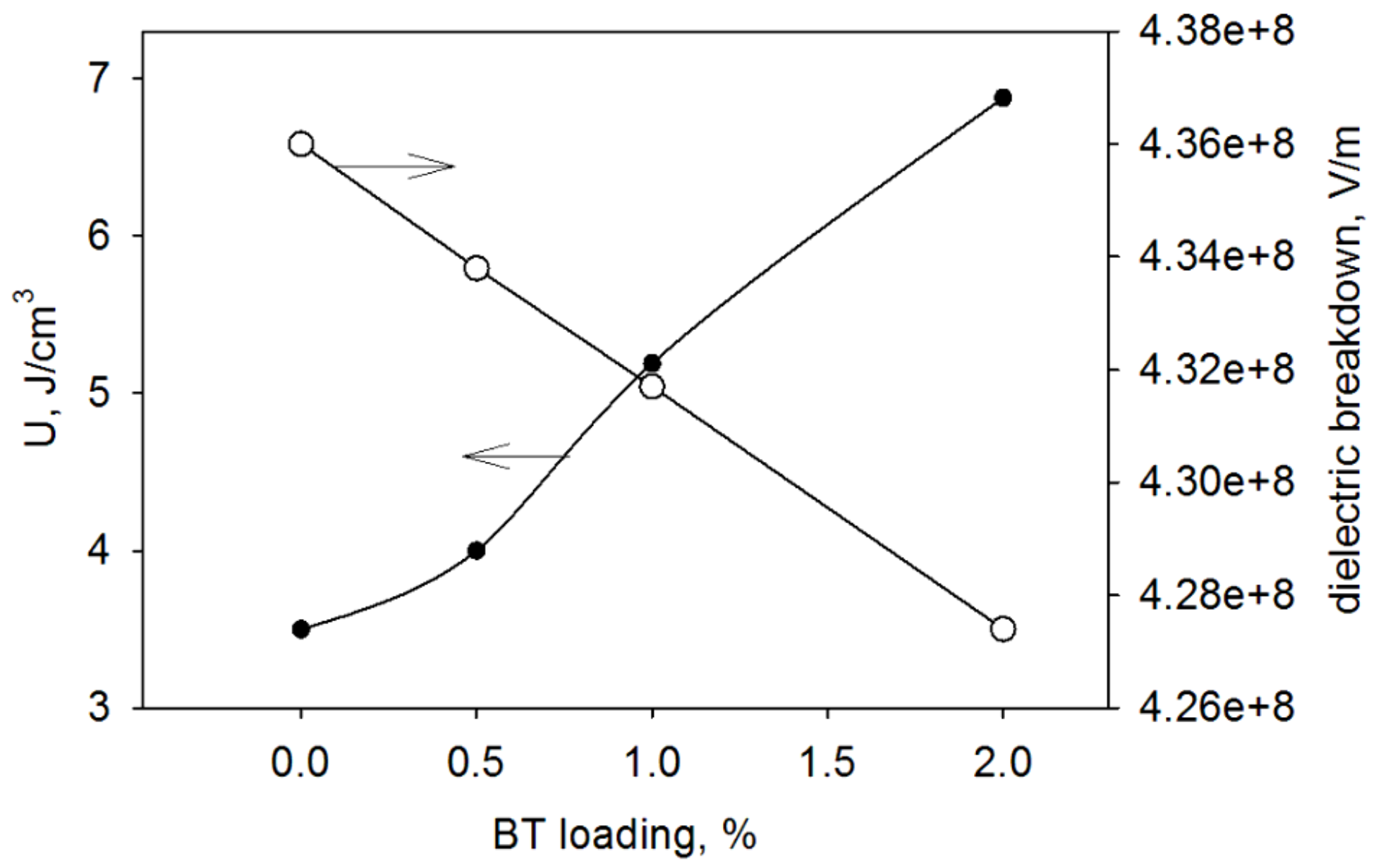

Figure 11

Electric energy density for neat HPC and HPC/BT composites as a function of BT loading 